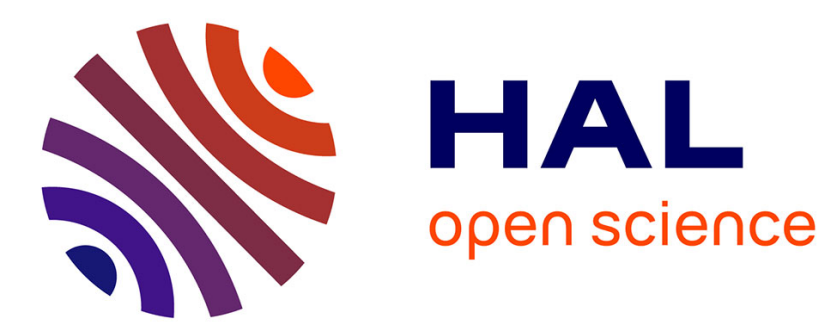

\title{
Le jugement des fléches
}

Paul Braffort

\section{To cite this version:}

Paul Braffort. Le jugement des fléches. Revue de Synthèse, 2009, 130 (1), pp.67-101. 10.1007/s11873009-0071-x . hal-00478460

\section{HAL Id: hal-00478460 https://hal.science/hal-00478460}

Submitted on 30 Apr 2010

HAL is a multi-disciplinary open access archive for the deposit and dissemination of scientific research documents, whether they are published or not. The documents may come from teaching and research institutions in France or abroad, or from public or private research centers.
L'archive ouverte pluridisciplinaire HAL, est destinée au dépôt et à la diffusion de documents scientifiques de niveau recherche, publiés ou non, émanant des établissements d'enseignement et de recherche français ou étrangers, des laboratoires publics ou privés. 


\title{
LE JUGEMENT DES FLÈCHES Un essai d'épistémologie appliquée
}

\author{
Paul BRAFFORT*
}

RÉSUMÉ: Diagrammes, schémas, itinéraires, nous sommes accoutumés aux formes sagittales de la représentation. Ces formes évoquent souvent des mouvements que l'écriture gèle nécessairement sur le papier alors qu'elles se proposent d'en exprimer la dynamique. Des progrès récents des techniques de communication et d'expression permettent parfois de surmonter ces difficultés. Lorsqu'elles peuvent se déployer dans l'espace (et le temps) d'un support susceptible d'entrer à son tour en mouvement (une machine - ou simplement une main), ces formes « libérées » permettent alors de lever (ou tout au moins d'ébranler) d'importants obstacles épistémologiques qui subsistent dans la pratique comme dans la théorie.

Mots-CLÉs: flèches, schémas, diagrammes de fluence.

\author{
THE JUDGMENT OF ARROWS \\ An essay on applied epistemology
}

ABSTRACT: Diagrams, schemata, itineraries, we are accustomed to forms of arrowheads in representation. These forms often evoke movements that writing necessarily freezes on the paper while they propose to themselves to express their dynamics. Recent progress in techniques of communication and of expression permit us at times to surmount these difficulties. When they can spread out into space (and time) of a prop able to entrer in its turn into movement (a machine - or simply a hand), this "liberated" forms permit us to remove (or at the every least to rattle) important epistemological obstacles which subsist in practice as in theory.

KEYWORDS: arrows, schemata, diagrams of fluency.

* Paul Braffort, né en 1923, est entré au Commissariat à l'énergie atomique en 1949 où il a créé et dirigé le laboratoire de calcul analogique. Détaché à EURATOM, de 1959 à 1963, puis à l'ESTEC (European Space Technology Centre) et à l'université de Paris XI (Orsay), il a été visiting scholar à l'université de Chicago, puis directeur de programme au Collège international de philosophie. Il a notamment publié Computer Programming and Formal Systems, en collaboration avec David Hirschberg (Amsterdam, North-Holland, 1963); L'Intelligence artificielle (Paris, Presses universitaires de France, 1968); Science et littérature: les deux cultures, dialogues et controverses pour l'an 2000 (Paris, Diderot, 1998).

Adresse: 7, rue Charles V, F-75004 Paris.

Courrier électronique: paul.braffort@noos.fr 
DAS URTEIL DER PFEILE

\section{Ein Versuch der angewandten Epistemologie}

Zusammenfassung: Diagramme, Schemata, Streckenpläne: wir sind an pfeilartige Repräsentationsformen gewohnt. Diese Formen erinnern häufig an Bewegungen, die die Schrift notwendigerweise auf das Papier fixiert und die sich anheischig machen, die Dynamik auszudrücken. Die jüngeren Fortschritte der Kommunikations- und Ausdruckstechniken erlauben bisweilen, diese Schwierigkeiten zu überwinden. Wenn sie sich im Raum (und der Zeit) eines Trägers entfalten können, der seinerseits in Bewegung geraten kann (eine Maschine - oder einfach eine Hand), erlauben diese „,befreiten“"Formen, wichtige epistemologische Hindernisse zu beheben (oder mehr oder weniger zu erschüttern), die in Praxis und Theorie gleichermaßen bestehen.

STICHWÖRTER: pfeile, schemata, flußdiagramme.

$$
\text { بول بر افور حُكْمُ الأسُهُم. رسالة في الابستيمولوجيا التطبيقيَّة }
$$

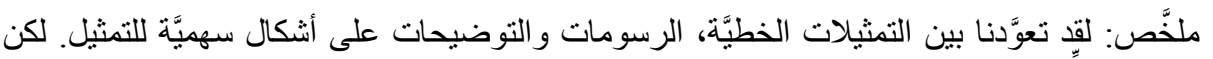

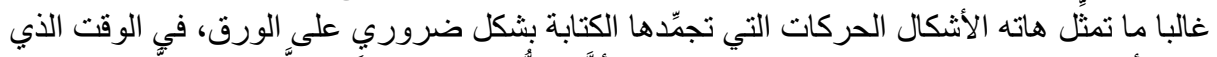

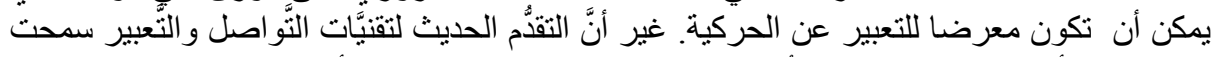

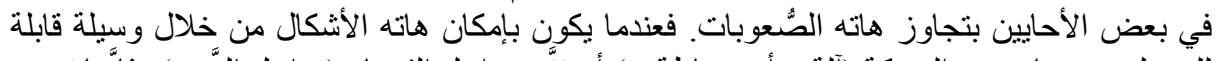

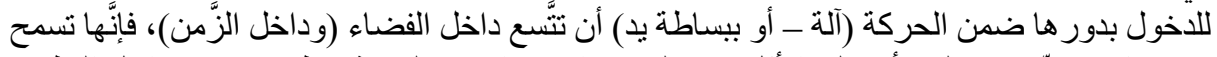

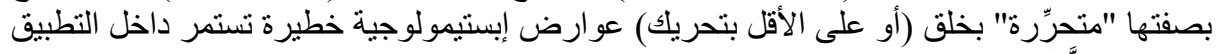
كما داخل النَّظرية. الكلمات المفاتيح: الأسهر، التمثيلات الخطبَّة، منحنيات التَّيُر.

矢の評価。応用認識論の試み

$$
\text { ポール・ブラフォール }
$$

要約：グラフ、図表、道順というように、我々は矢の形の象徵に慣れてい る。これらの形は、この形自体が活力を表現しようとするにもかかわらず、 紙上で書く場合、書くという行為を必然的にコントロールしなければならな いという特徵を持ち合わせている。この形が、動くことが可能である（機 械、もしくは単に手のような）支柱の空間（そして時間）の中で広がって行 くとき、実践、且つ理論分野に存在している多くの認識学的困難を取り除く ことができる。

キーワード : 矢、眓表、流量グラフ 
$\mathrm{L}^{\prime}$ ambiguïté que l'on peut percevoir dans le titre de cet essai devrait être fructueuse. Car lorsqu'on distingue un jugement établi grâce à l'utilisation de flèches de celui que l'on pourrait porter sur les flèches elles-mêmes, on rend explicite déjà une direction, un mouvement, une intention dans le processus même du jugement, et peutêtre un projet épistémologique.

Depuis Zénon, l'image de la flèche a trouvé de très nombreux emplois dans les domaines les plus divers. On en présentera plus loin quelques exemples: elle apparaît déjà métaphoriquement dans la dialectique telle que Zénon lui-même l'a pratiquée, puis comme symbole graphique dans l'expression de nombreux schémas et diagrammes, dans la conceptualisation des domaines les plus variés de la science et de la technique et même dans certains des aspects de la vie quotidienne, tels que les divers modes de la signalisation, routière, urbaine, et même domestique. Dès que cela a été techniquement possible, des représentations graphiques du concept de flèche ont été introduites dans les textes pédagogiques et dans les exposés scientifiques, en utilisant une grande variété de graphismes. On peut d'ailleurs découvrir désormais la plupart de ces graphismes et les exploiter dans des sites Internet spécialisés ${ }^{1}$. En voici un exemple, emprunté au peintre Ludovic Sauvage.

Si les flèches précisent une direction, elles ne prononcent pourtant explicitement aucun verdict qui sera, lui, déterminé par le contexte (qui n'éliminera pas toujours l'ambiguïté, d'ailleurs).

On remarquera aussi qu'une ambiguïté seconde peut apparaître lorsqu'on évoque le sens même des flèches dans les textes - ou les illustrations - où elles figurent: car la signification que l'on associe naturellement au mot «sens»

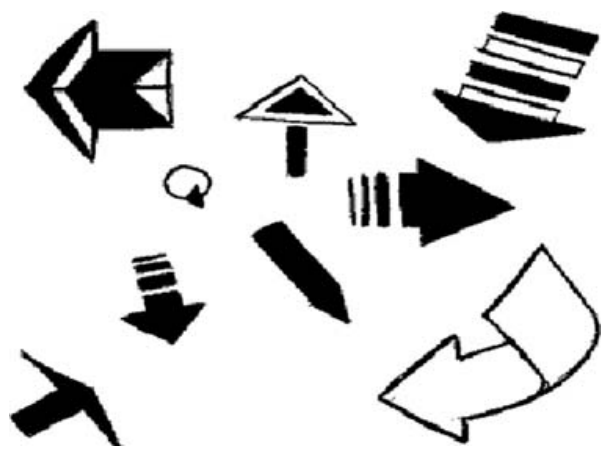
peut être précisément signification mais aussi bien direction. Ici encore le contexte peut éventuellement trancher.

Le titre de cet essai fait évidemment allusion au film de Samuel Fuller (tourné en 1957), un superbe « western » dont le titre, en version originale, est Run of the arrow (course de la flèche). La traduction littérale française introduit alors une autre ambiguiité : car le mot «course », en français, peut être interprété (entre autres possibilités) comme trajet ou comme déplacement, ce qui introduit une composante dynamique que les formes graphiques ne peuvent pas toujours bien représenter.

C'est précisément l'objectif principal, ici, de faire apparaître, en étudiant et en comparant les différentes utilisations du concept de flèche, la dialectique entre statique et dynamique dans l'expression, la représentation des situations et des phénomènes. La comparaison portera, autant que possible, sur les aspects les plus divers du processus

1. Voir en particulier: http://www.icomania.com; http://Fbreuill.chez.com/html/Fl_ches.html; http://www.decomania.org/gifomania/fleches/page1.htm. Dans ce dernier cas, les flèches produites sont animées. Parfois tridimensionnelles, elles scintillent, clignotent, s'inversent, etc., témoignant d'une dynamique qu'il sera nécessaire d'expliciter et d'analyser. 
de la représentation. Les flèches auront alors, suivant les cas, un simple rôle heuristique ou assumeront au contraire un rôle réellement performatif.

Mais un obstacle demeurera - au moins lors d'une lecture «traditionnelle de ce texte »- celui que constitue la contrainte de la lecture (après celle de l'écriture) sur un support nécessairement immobile. Il en ressortira peut-être le désir de lever cet obstacle - au moins de contribuer à le faire.

\section{PETITE TAXINOMIE SAGITTALE}

Avec un arc ou avec une sarbacane, les flèches ont été (et sont encore) des armes de combat. La culture populaire en a conservé le souvenir dans la métaphore de la flèche du Parthe. Mais à la notion de flèche sont souvent associées celles de trajet, de direction, de vitesse. Il n'est donc pas étonnant que cette notion ait été utilisée très tôt au service des formes sophistiquées de la dialectique. Dans la rhétorique de Zénon, elle joue un rôle essentiel dans les quatre arguments majeurs de l'Éléate, et en particulier dans le second argument, qui est précisément celui de la flèche ainsi que le rappelle Léon Robin:

« Une chose étant toujours dans un lieu égale à elle-même, la flèche du vol ne sort pas, dans un instant indivisible, du lieu même qu'elle occupe; ne changeant pas de lieu, elle est donc immobile, et il en sera toujours de même si le temps est, par hypothèse, une somme d'instants ${ }^{2}$.»

Léon Robin présente ensuite le quatrième argument, celui du stade et il l'accompagne d'une illustration, ce qui est exceptionnel dans ce type d'ouvrage, et qui représente trois groupes parallèles de quatre masses indivisibles ou points.

Il évoque alors leur mouvement - ou leur absence de mouvement - dans un stade afin de montrer l'inintelligibilité du mouvement. Pourtant, pour commode et naturelle qu'elle soit, l'utilisation de la forme graphique de la flèche pour la représentation et l'argumentation est relativement tardive. L'importance du concept de relation est certes bien comprise dès le Moyen Âge, mais son expression graphique fait souvent usage d'images complexes où l'orientation du flux n'est pas clairement indiquée. Ce sont d'ailleurs les formes arborescentes qui sont privilégiées et, là encore, l'orientation des arcs demeure implicite.

Chez Raymond Lulle (1235-1315), les arbres constituent un outil privilégié de l'expression des relations, principalement des relations hiérarchiques: son Arbre de la science est composé de quatorze arbres spécifiques exprimant des relations de précédence ou d'antériorité .

2. RoBIN, 1923, p. 113.

3. Voir Johnson, 1987. 
On remarquera, dans les figures que Lulle nous propose, le double mouvement (vers le haut ou vers le bas), des branches et des racines: déploiement des connaissances et recherche des sources profondes de la connaissance.

Il faut attendre trois siècles pour que Ramus (Pierre de la Ramée, 1515-1572), humaniste et professeur au Collège de Presles, construise à son tour des arborescences particulières: ce sont des dichotomies ${ }^{4}$. Ces arborescences se présentent horizontalement, contrairement à celles de Lulle (mais l'orientation demeure implicite). L'intention, ici, est essentiellement pédagogique. Page suivante, on présente un exemple, datant de 1555 , où la vie de Cicéron est décrite - et même organisée - comme projection du curriculum des

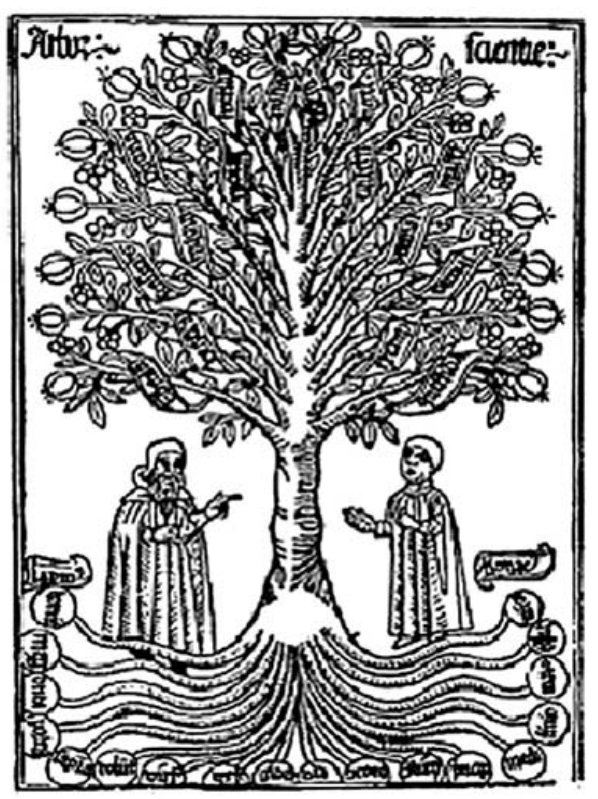
arts; sans doute le premier exemple de biographie schématisée géométriquement.

On notera avec intérêt que dans son ouvrage majeur ${ }^{5}$, Walter Ong évoque Ramus (et le « ramisme ») dans un chapitre intitulé Typographic Rhapsody. Le mot « rhapsodie» mérite d'être souligné: nous retrouverons des convergences «plurimodales » au cours de notre étude.

Dans un travail récent, Jeffrey Nicholson a entrepris une analyse très complète de l'utilisation des diagrammes dans la littérature technique contemporaine ${ }^{6}$. Il a analysé 495 publications contenant 1899 figures et élaboré une intéressante classification (il inclut, dans le concept de « figure », les tableaux, les cartes, les figures, etc., aussi bien que les diagrammes sagittaux). Une difficulté qu'il signale, dans son travail de classification, est « [...] the problem of understanding the meaning of an arrow ». Il énumère alors diverses acceptions possibles:

1) L'étape suivante d'un projet;

2) L'instruction suivante dans un programme;

3) Le mouvement d'un flux de données;

4) La transmission d'un message;

5) L'influence;

6) La cause;

7) Le déplacement dans un espace;

8) La direction d'une relation abstraite;

9) Quelque chose qu'il est important de regarder.

4. On doit la redécouverte de Ramus à Frances Yates et Walter Ong (voir YATES, 1975; ONG, 1958).

5. ONG, 1977.

6. Nicholson, 2005. 


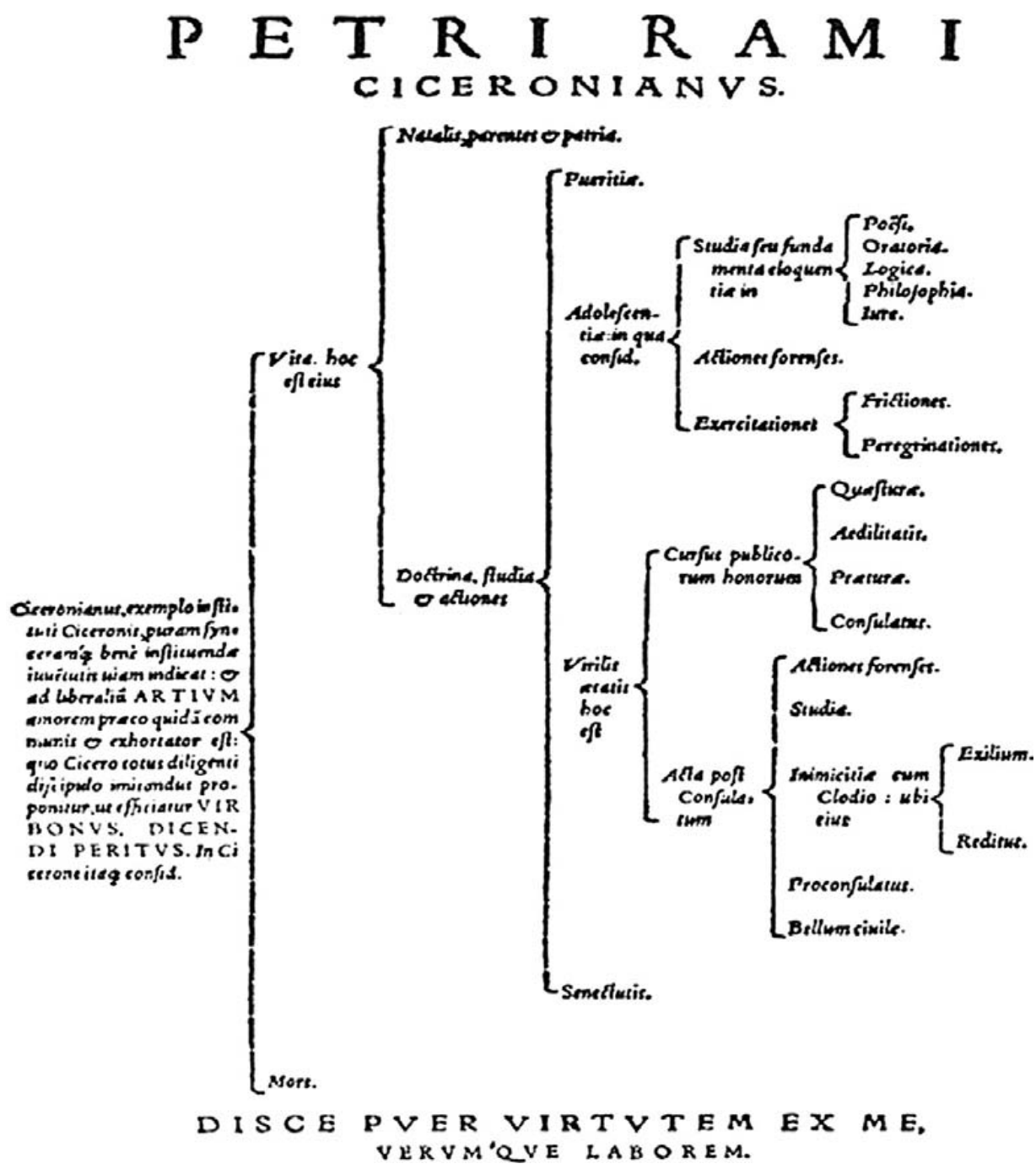

Dichotomies, «polychotomies », schémas, organigrammes, etc., l'expression de ces structures d'organisation demeure essentiellement typographique et ne fait usage d'aucun symbole nouveau (à l'exception des « accolades » introduites par Ramus). Les flèches ne sont encore, le plus souvent, qu'implicites, comme pour les arbres de Lulle, et ceci jusqu'à une date récente.

Les sciences, dès la Renaissance, et en particulier la mécanique, l'optique et plus généralement la physique en ont pourtant l'emploi, mais il faudra attendre Galilée et même Newton pour qu'elles apparaissent dans les textes. C'est la notation vectorielle des forces qui intervient alors, avec leurs fameux triangles et parallélogrammes. 
Les progrès rapides de la physique théorique à la fin du XIX ${ }^{\mathrm{e}}$ siècle imposent ensuite, après William Hamilton (1805-1865), Willard Gibbs (1839-1903) et Oliver Heaviside (1850-1925), la prise en compte des formes vectorielles: le champ électrique et le champ magnétique doivent être représentés par des vecteurs, mais les équations aux dérivées partielles qui en déterminent l'évolution sont encore souvent présentées sous une forme "développée » où les trois composantes sont simultanément écrites (sans flèche). Souvent, c'est la police typographique utilisée (caractères gras en général) qui permet de distinguer l'entité vectorielle d'une entité scalaire. Les flèches ne sont donc pas encore inscrites dans le texte, mais elles sont parfois « épifixées » au-dessus des expressions de certains opérateurs comme le gradient ou le rotationnel.

On pourrait s'attendre à ce qu'avec le grand essor de la logique mathématique, au début $\mathrm{du} \mathrm{xx}^{\mathrm{e}}$ siècle, une abondance de flèches de toutes sortes fasse son apparition. Il n'en est rien, même chez des auteurs pourtant généreux en innovations notationnelles comme le sont Russell et Whitehead (qui utilisent pourtant, à quelques reprises, des flèches épifixées et des flèches verticales plus ou moins tronquées) ${ }^{7}$. Les logiciens polonais de Varsovie et de Cracovie, tels que Lukasiewicz, Lesniewski ou Chwistek se livrent bien à une débauche d'imagination notationnelle pour leurs primitives, mais l'implication, par exemple, est le plus souvent représentée par un C (ou même par le symbole $\supset)^{8}$.

La présentation (ou représentation) des réactions chimiques sous forme symbolique s'inspirant de l'algèbre pourrait passer naturellement, par l'utilisation de la flèche $\rightarrow$ qui impliquerait alors une transformation matérielle. Dans le cas des transformations réversibles, on pourrait alors utiliser $\leftrightarrow$. Mais pendant longtemps, on appellera ces expressions des équations et on utilisera donc plutôt ${ }^{9}$ le symbole de l'égalité « $=$ ». Plus tard, les techniques de représentation relationnelles s'affineront en chimie - et, singulièrement, en chimie organique.

Dans Les Affinités électives (1809), Goethe construit bien l'intrigue sur le modèle d'une réaction chimique à quatre composants, telle que:

$$
\text { Chaux }+ \text { Acide sulfurique } \rightarrow \text { Gypse }+ \text { Eau gazeuse }
$$

Mais il s'abstient, toutefois, de proposer une notation spécifique ${ }^{10}$.

Par contre, les applications les plus diverses apparaissent peu à peu, qui mettent en jeu le spiritualisme, avec les «arbres séphirotiques» dans leurs différentes variantes ou - pour des utilisations plus actuelles - traduisent une évolution temporelle, et la complexité de certaines situations politiques dans une élection présidentielle

7. Russell et Whitehead, 1910. Les flèches horizontales $\rightarrow$ et $\leftarrow$ sont définies en 32.01 et 32.02 et même, en 35.03 et 35.04 , les flèches verticales $\uparrow$ et $\downarrow$ que l'on retrouvera en informatique.

8. On notera la discrétion du Dictionary of Symbols of Mathematical Logic de Robert Feys et Frederic B. Fitch. Une notation sagittale n'apparaît qu'en 12.2 (FEYs et FITCH, 1969, p. 31). Elle y symbolise l'implication.

9. Comme ce sera aussi le cas, d'ailleurs, dans les débuts de l'informatique.

10. BRAFFORT, 1999, p. 86. 
qu'illustrent l'abondance et le fouillis des flèches, comme ci-contre.

Le plus souvent, cependant, c'est bien l'idée d'une transformation ou d'une évolution qui est évoquée, transformation du contenu ou de la forme.

Ainsi, l'encadré page suivante ${ }^{11}$ illustre une série de transformations de formes qui accompagnent nécessairement, en les codifiant, des transformations de contenus et l'on pourra noter que les contenus eux-mêmes peuvent se lire comme des codifications. Ce sont donc des transductions que l'on trouve associées, dans le schéma lui-même, à ces domaines distincts mais comparables - et

Official Florida Presidential Ballot

Follow the arrow and Punch the appropriate dot.

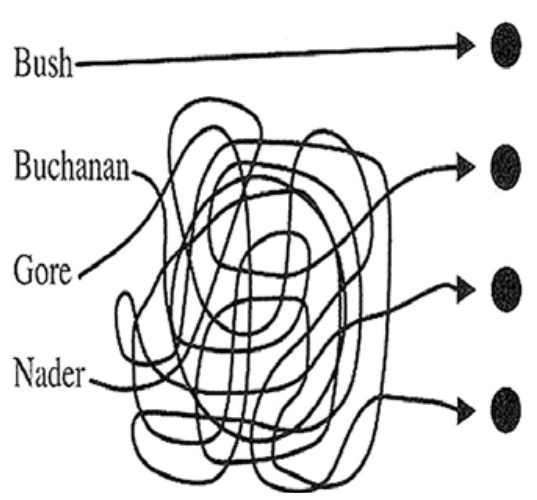
parfois homologues - que sont ceux de la linguistique et de la génétique moléculaire (et, pourquoi pas, de la cryptographie).

On remarquera que les trois flèches qui figurent dans le tableau n'ont pas exactement la même signification - ni la même portée: on y constate la grande polysémie du symbolisme. La première flèche explicite un processus de traduction (d'un système de notation dans un autre). La suivante présente un autre processus (dont l'algorithme est précisé dans l'encadré). La troisième annonce le résultat de l'encodage annoncé. On voit clairement ici à l'œuvre le couplage de la succession temporelle d'actions transformatrices et de l'enchaînement logique de transformations (formelles ou non): relations entre flèches, qui pourraient elles-mêmes être symbolisées par des flèches.

Ici la diversité des formes et des contenus est exploitée par Eduardo Kac pour porter un message qui est délibérément épistémologique.

\section{DE LA PRATIQUE DES FLÈCHES À UNE THÉORIE DES FLÈCHES}

$\mathrm{Au}$ cours $\mathrm{du} \mathrm{Xx}^{\mathrm{e}}$ siècle, de nombreux domaines d'activité se sont successivement emparés du symbole de la flèche qui est finalement devenue un outil de représentation, une forme d'expression privilégiée dans de nombreux domaines et, en premier lieu, dans les divers secteurs de la science, en particulier de la physique: ces secteurs sont ceux qui précisent une dynamique dans l'interprétation des phénomènes ou qui en expriment l'orientation. Les catégories du temps et de l'espace sont ainsi sollicitées et parfois même leur couplage.

Avec la mécanique rationnelle et sa dynamique, c'est évidemment l'électromagnétisme qui en fait un usage essentiel, dans la phase initiale de formalisation qui va de Faraday à Maxwell. Les lignes de forces sous-tendent des champs de vecteurs qui leur sont tangents. D'autres schémas permettraient de «matérialiser» les flux et les

11. KAC, 1999. 
Let man have dominion over the fish of the sea and over the fowl of the air and over every living thing that moves upon the earth

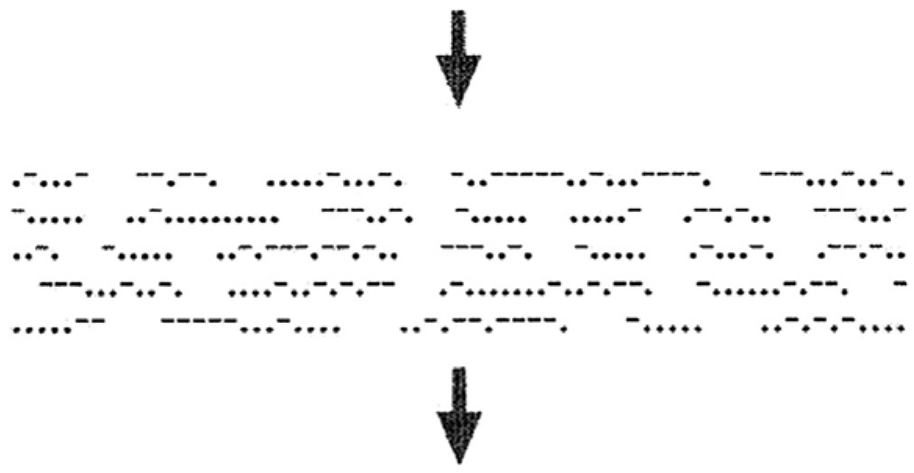

\section{Morse to DNA conversion principle}
DASH (-) $=\mathrm{T}$
$A=$ WORD SPACE
DOT $()=.\mathrm{C}$
$\mathrm{G}=$ LETTER SPACE

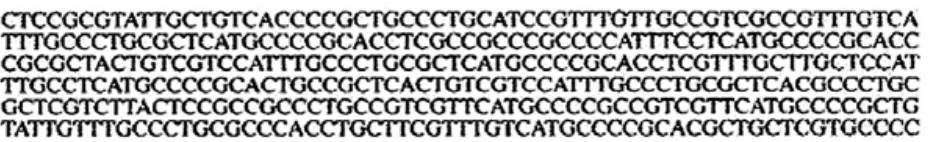

tensions de l'hydrodynamique. Nous en donnons un exemple, à la page suivante, où l'esprit de Faraday est déjà manifeste (les flèches désignent ici l'orientation du moment magnétique; voir l'illustration en haut de la page 76).

L'expression mathématique des lois du mouvement pour ces différents phénomènes met en œuvre la notion de « champ de vecteurs » qui peuvent donc être représentés par des flèches et ne posent pas encore de problème épistémologique. Mais une situation nouvelle apparaît dès que la thermodynamique prend son essor et que surgit le problème fondamental qu'exprime le second principe: celui de la compréhension des phénomènes dans leur irréversibilité; le problème, encore très sensible aujourd'hui, de la flèche du temps. 
Cette flèche bien spécifique n'est pas qu'une figure de rhétorique. Elle illustre d'ailleurs, comme c'est assez naturel, de nombreux schémas destinés à expliciter quelques aspects essentiels de la relativité restreinte ${ }^{12}$.

Électromagnétisme et thermodynamique ne font pas le même usage de la flèche temporelle. Mais ces deux aspects se croisent nécessairement lorsqu'on examine de près, dans leur aspect temporel, les solutions des équations fondamentales qui déterminent le champ.

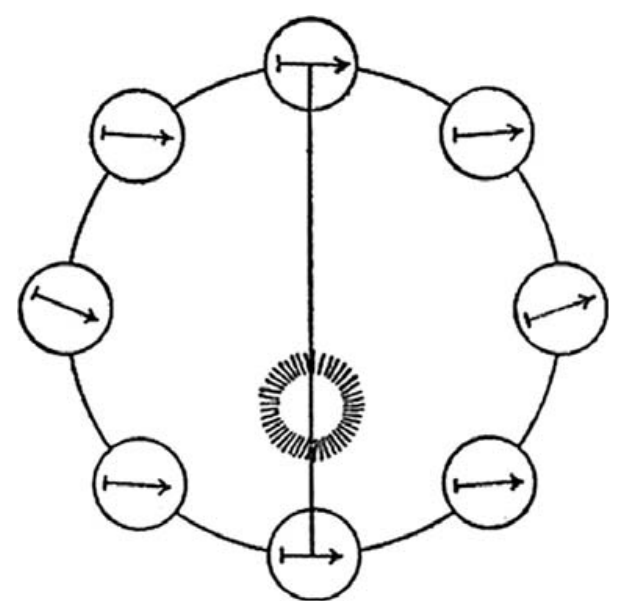

186,000 MILES PER SECOND
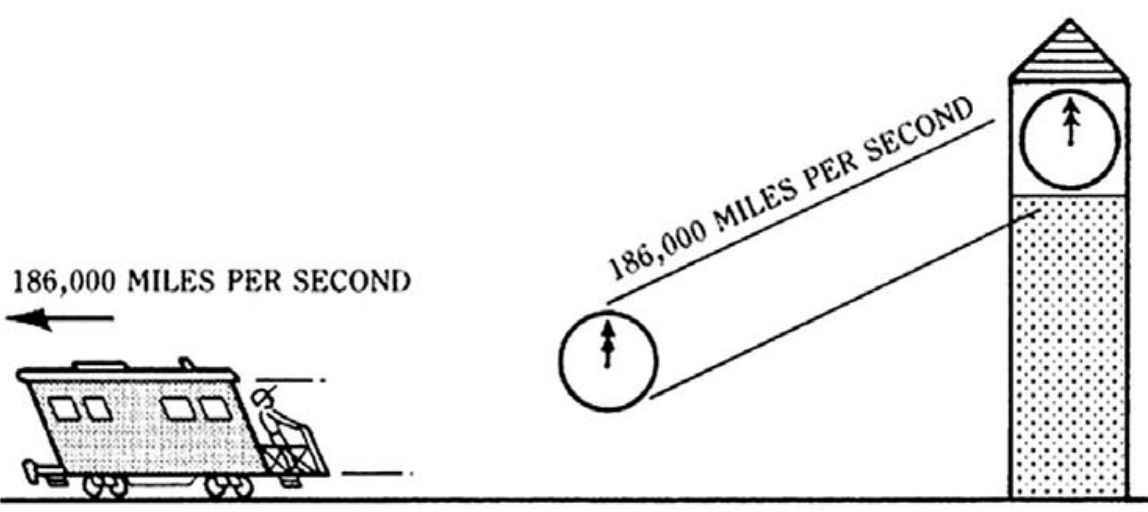

A train moving away from a clock tower at the speed of light. After a minute passes, the observer on the train notes that the time on the clock remains 12:00 o'clock.

L'aspect « cinématique » et l'aspect «thermodynamique » interfèrent, de façon particulièrement intrigante, dans l'électrodynamique imaginée par Wheeler et Feynman. Voulant éliminer les « énergies infinies » de la théorie qui apparaissent dans l'électrodynamique classique de Lorentz, ils observent que les solutions des équations

12. Schlain, 1991, p. 124. 
du champ peuvent être aussi bien avancées (en $t+x / c)$ que retardées (en $t-x / c)$ et utilisent à la place la demi-somme des effets avancés et des effets retardés. Ils éliminent ensuite l'effet paradoxal des effets avancés en considérant l'univers comme un absorbeur où les actions avancées interfèrent pour s'éliminer mutuellement ${ }^{13}$. Le schéma ci-dessous explicite (mais seulement avec l'explication détaillée du texte) leur raisonnement:

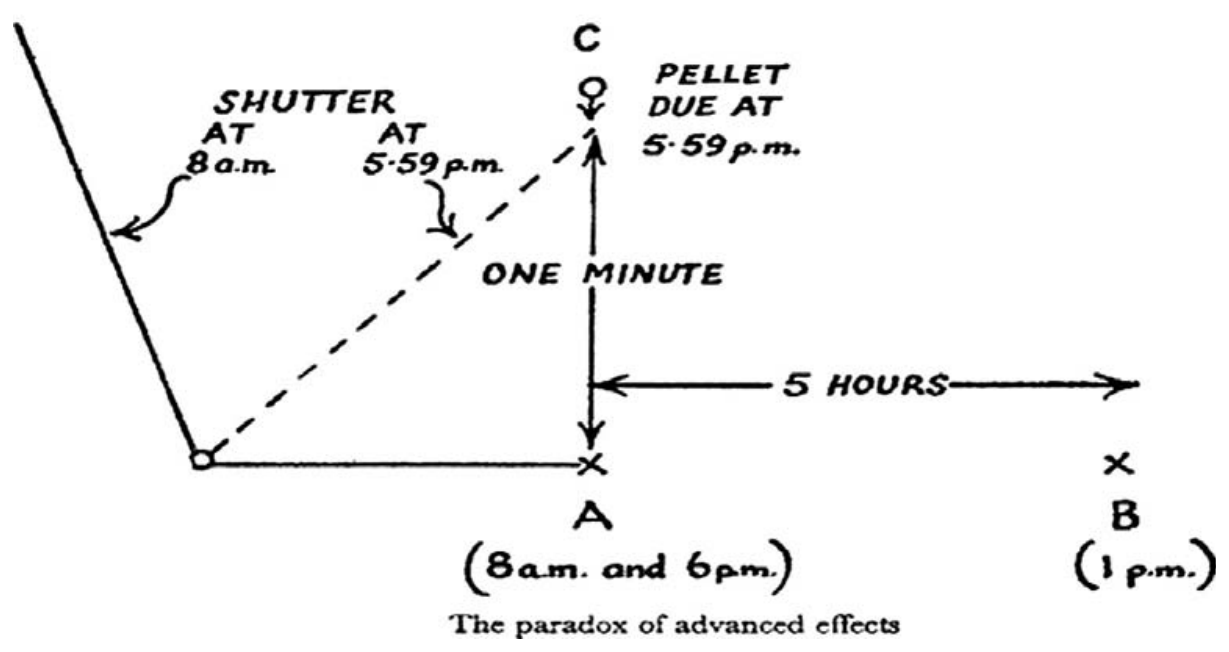

De présentation et d'interprétation délicate, la théorie n'eut qu'un succès limité, mais Feynman en conserva l'idée essentielle: considérer les antiparticules comme des particules voyageant «à rebrousse temps », une idée qu'il utilisera dans ses fameux diagrammes en électrodynamique quantique. Dans le schéma inséré page suivante, la dimension verticale est celle de l'espace, l'horizontale celle du temps ${ }^{14}$.

L'existence d'actions avancées pouvait donner lieu à des paradoxes logiques ou physiques que Wheeler et Feynman se sont efforcés d'exorciser. Mais ces «actions avancées " peuvent aussi jouer un rôle dans l'interprétation du deuxième principe de la thermodynamique, comme le suggère une note récente de Landsberg et Vickers ${ }^{15}$.

Les deux publications fondamentales de Wheeler et Feynman parurent respectivement en 1945 et 1949, alors que le premier exposé de la théorie avait été présenté à Princeton, devant un parterre prestigieux (Einstein, Neumann, Wigner, Pauli, etc.), en février 1941. Les flèches que l'on rencontre dans les théories sont inscrites dans l'espace et dans le temps (avec, dans l'avant-dernière figure, un rôle indiciel ou logique).

13. Wheeler et Feynman, 1949, p. 425. Le paradoxe a été clairement exposé par Landsberg, 2000. Voir aussi Cramer, 1983.

14. Cet épisode passionnant de l'histoire de l'électrodynamique a été traité de façon approfondie dans la biographie de MeHra, 1994, particulièrement p. 92 sqq.

15. LANDSBERG, 2000. 


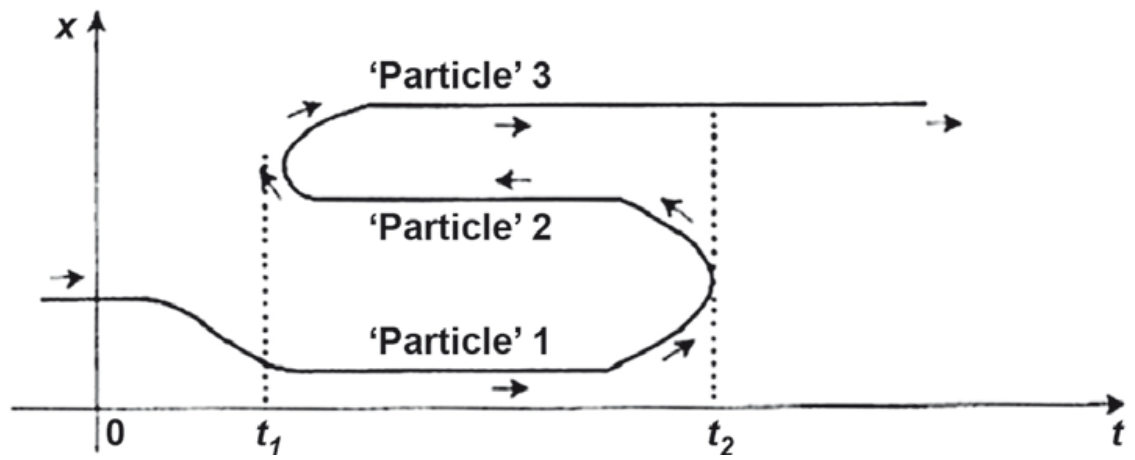

La trajectoire d'une particule fait demi-tour au temps $t_{2}$, puis à nouveau au temps $t_{p}$, se trouvant dans la direction croissante du temps. L'observateur verra trois particules dans la période de temps qui sépare $t_{1}$ et $t_{2}$.

Or c'est précisément à la même époque, en 1945 (et, au moins en partie, à Princeton), que naît une nouvelle discipline mathématique appelée à un grand avenir: la théorie des catégories. Il s'agissait, pour Eilenberg et Mac Lane, d'introduire des objets algébriques (tels que les groupes d'homologie) dans l'étude des espaces topologiques. Il s'agissait plus précisément d'étudier l'opération qui consiste à « appliquer » un espace sur un autre à l'aide d'une fonction continue et d'étudier les effets de cette opération sur les groupes correspondants ${ }^{16}$. C'est l'époque où, sous l'influence de grands algébristes comme Van der Waerden et surtout du groupe très actif de Bourbaki, animé par André Weil, Henri Cartan et d'autres, le concept de base en mathématique est celui d'ensemble, avec les concepts associés d'élément et de partie. D'après Bourbaki, le concept d'ensemble est le fondement de tous les objets mathématiques.

Les relations binaires (qu'incarnent précisément les flèches) n'apparaissent pas dans les "structures fondamentales de l'analyse » telle que Bourbaki les définit; il faudra attendre pour cela Jacques Riguet et Claude Berge pour qu'elles soient intégrées, de façon marginale, cependant. Mais des relations binaires particulières seront utilisées: projections, isomorphismes (et autres morphismes) comme outils d'analyse et d'évaluation.

Avec la théorie des catégories ce sont d'autres concepts qui apparaissent au premier plan et qui ne sont pas dénués de parenté, bien entendu, avec ceux qui s'imposèrent dans la théorie des ensembles: ceux d'objet, de foncteur ${ }^{17}$ et de flèche. À la notion même d'ensemble, se substitue, d'une certaine façon, celle de catégorie. Tous ces concepts nouveaux - y compris celui de flèche - peuvent constituer eux-mêmes des catégories. Et, tout comme il existe une catégorie des groupes, une catégorie des flèches, il existe une «catégorie des catégories » qui ne donne pas prise aux célèbres paradoxes de la théorie des ensembles.

16. L'article fondateur est, de l'avis général, EILENBerg et MAC LANE, 1945.

17. J'utilise ici une typographie devenu standard en algèbre des catégories. 
Dans sa récente thèse de doctorat ${ }^{18}$, Franck Jedrzejewski s'est explicitement inspiré de l'essor quasi simultané des techniques de calcul diagrammatiques «à la Feynman » et de la théorie mathématique des catégories pour effectuer une revue systématique de l'usage des diagrammes dans la science et dans la philosophie des sciences. La grande technicité de certains développements (notamment en ce qui concerne l'algèbre des catégories) rend difficile une appréciation pertinente de l'entreprise: la distance est considérable entre les arbres de Lulle, les carrés sémiotiques de Greimas (issus des carrés d'Apulée) et les schémas fonctoriels de Grothendieck. Comme il est fréquent dans la mathématique contemporaine, l'utilisation de mots usuels pour désigner des objets mathématiques sophistiqués (ensemble, catégorie, filtre, immeuble, faisceau, etc.) nous incite parfois au glissement métaphorique. La notion de «problème universel », en est un exemple.

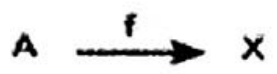

Catágorie $A$

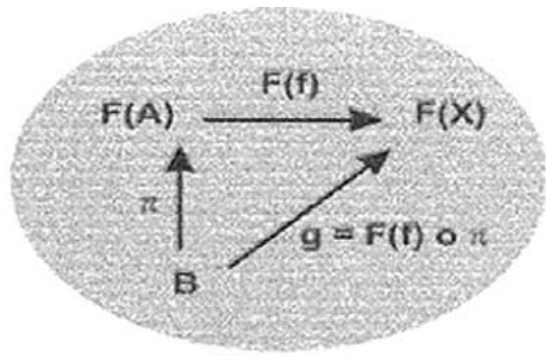

Catégorie ' $B$

Cependant Jedrzejewski met l'accent sur un aspect des diagrammes que l'expression iconique implique, mais que le texte nécessairement en «langue naturelle» ne peut aisément traduire: l'aspect spécifique du mouvement. Seule la flèche peut effectivement en faire pressentir l'immanence, comme c'est le cas avec l'illustration reprise ci-dessus. Évoquant «gestes et orientations diagrammatiques » et «machinerie diagrammatique », il déclare alors: «Comprendre, c'est assimiler un geste, être capable de le reproduire et de le prolonger ${ }^{19}$. » Puis il ajoute:

« [...] le moteur du foncteur est la machinerie diagrammatique, le noyau qui permet au diagramme de fonctionner et de produire une signification. Ce moteur qui confère au diagramme son mode de fonctionnement a un pouvoir d'engendrement plus important encore. C'est lui qui fait fleurir le geste et déploie ses virtualités ${ }^{20}$. »

18. JEDRZEJEWSKI, 2007

19. JedrzeJewski, 2007, p. 23. Et il cite à ce propos Jean Cavaillès dans Méthode axiomatique et formalisme. III La non-contradiction de l'arithmétique (Paris, Hermann, 1938, p. 178): « [...] comprendre [le calcul desarguien ou le procédé de la diagonale] est en attraper le geste, et pouvoir continuer. »)

20. JeDRZEJEWSKI, 2007, p. 28. 
La première (et la plus riche) des machineries, qui permet l'expression - et l'inscription - de notre pensée et de nos sentiments, c'est évidemment celle du langage: celle qui est à l'œuvre ici même, dans ces pages, comme dans celles que nous consultons, que nous écoutons, que nous analysons; discours, images ou textes.

Mais les textes, en particulier les textes théoriques, scientifiques ou épistémologiques, et plus spécialement ceux qui relèvent de la physique ou de la mathématique, utilisent une langue un peu différente de la langue naturelle, celle de la littérature ou de la communication générale; ils utilisent un langage augmenté, riche de signes où de symboles supplémentaire. La flèche est évidemment l'un de ces symboles.

Ce n'est que dans la première moitié $\mathrm{du} \mathrm{Xx}^{\mathrm{e}}$ siècle, que l'analyse linguistique se déploie vraiment. L'analyse syntaxique, en particulier, fait usage de plus en plus d'outils symboliques et formels, adoptant des formes de représentation graphiques efficaces, notamment pour l'expression des liens syntaxiques. On connaît dans ce domaine, bien entendu, les graphes de Chomsky, mais ils furent précédés dans ce domaine par les stemmas de Tesnière qui étaient essentiellement des arborescences. On trouve aussi des graphes orientés, et donc des flèches, à la même époque, dans l'expression des formules logiques du calcul des propositions ${ }^{21}$.

Mais les relations de nature sémantique peuvent recevoir aussi une représentation sagittale. En voici un exemple classique (relatif à un texte qui, comme souvent alors,

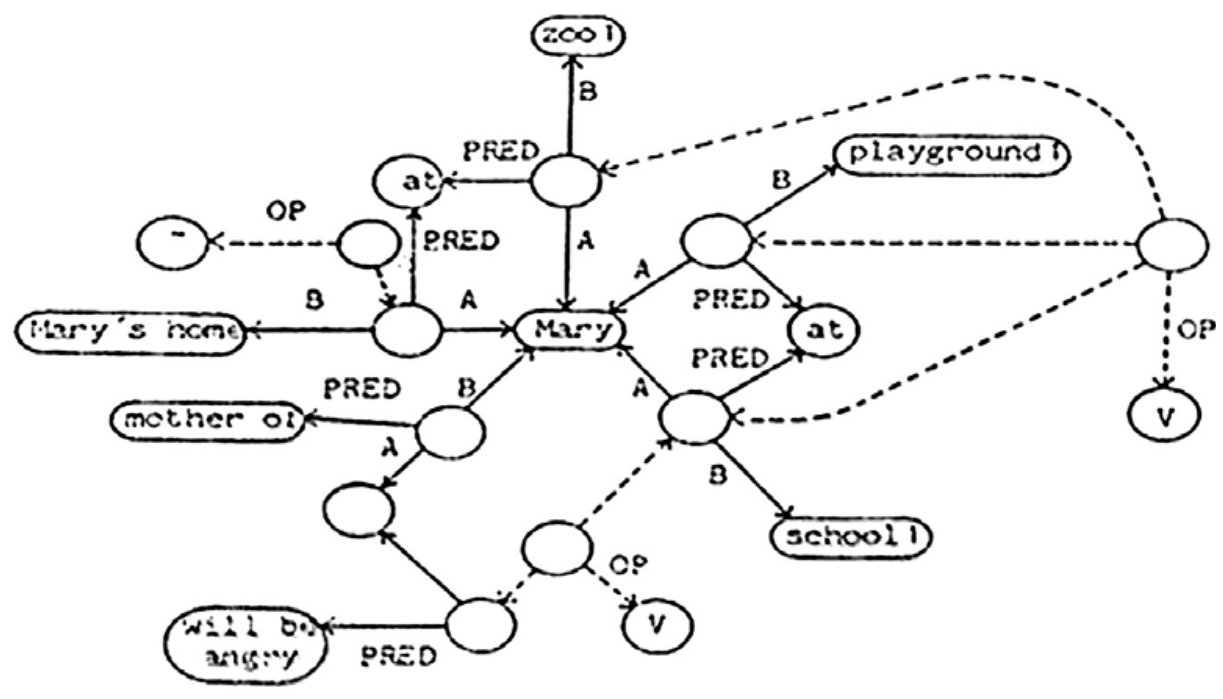

"Mary is not at rone: site is eitrer at school, or on the piajs: ourd, or at the zoo; if she is not at school. hor mother wili be arger"

21. Suszko, 1958, p. 213. 
témoigne d'une grande pauvreté de contenu !). L'idée d'une représentation diagrammatique des connections sémantiques est ancienne ${ }^{22}$.

La transformation d'un texte rédigé en langage naturel en un schéma (ou une suite de schémas) est, bien entendu, un processus complexe dont voici un autre exemple ${ }^{23}$. Le texte analysé est un fragment de l'autobiographie poétique de Jean Queval: Tout le monde descend ${ }^{24}$. Les étapes de l'analyse sont alors: 1) l'analyse lexicale et découpage en unité syntagmatiques; 2) l'analyse des syntagmes; 3) la résolution des anaphores; 4) la constitution de blocs sémantiques (dans l'esprit de la figure précédente). Voici le schéma qui condense ce passage du récit de Queval:

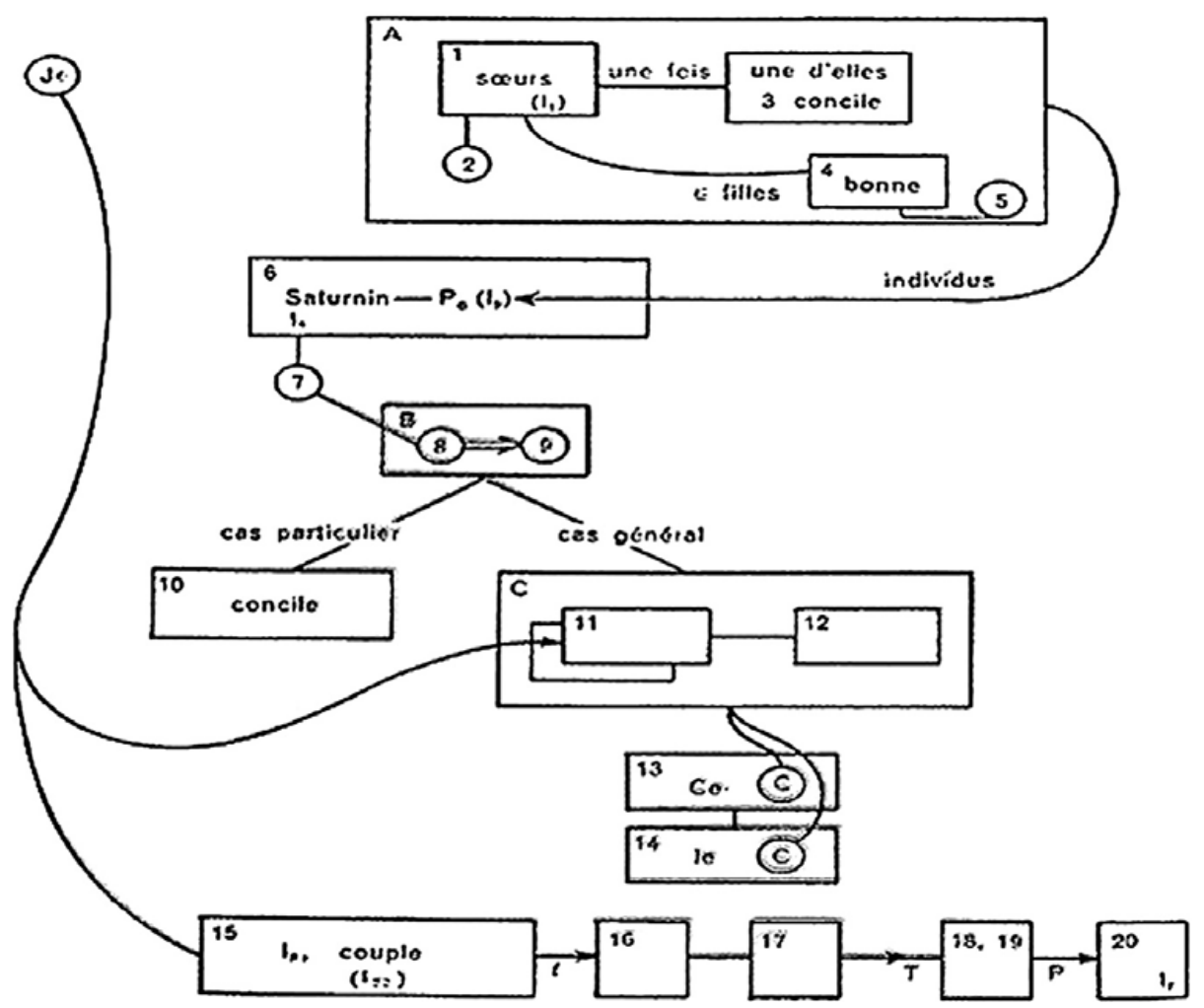

Il me semble intéressant de rapprocher le schéma ci-dessus, résultat d'une analyse qui n'est que partiellement algorithmique et qui contient donc une indéniable part

22. BrafFort et Leroy, 1959, p. 383. Depuis les exemples se sont multipliés comme en témoigne Roger Schank, Douglas Lenat, John Sowa, François Rastier, etc. Voir en particulier Beaugrande et DRESSLER, 1983.

23. BRAFFORT, 1968, p.114.

24. Jean Queval, Tout le monde descend, Paris, Mercure de France, 1959 et Plein Chant, 1988. Dans cette dernière édition, le passage analysé se trouve aux pages $46-48$. 
d'arbitraire et de subjectivité, de la planche suivante due à Chris Ware ${ }^{25}$. Ce fragment de comics, dépourvu de phylactère, relate un épisode des aventures de deux personnages, « Quimby », la souris et « Sparky », le chat (réduit à une simple tête), donne une bonne idée de la diversité et de la richesse de l'emploi des flèches pour une représentation cinétique et logique des événements.

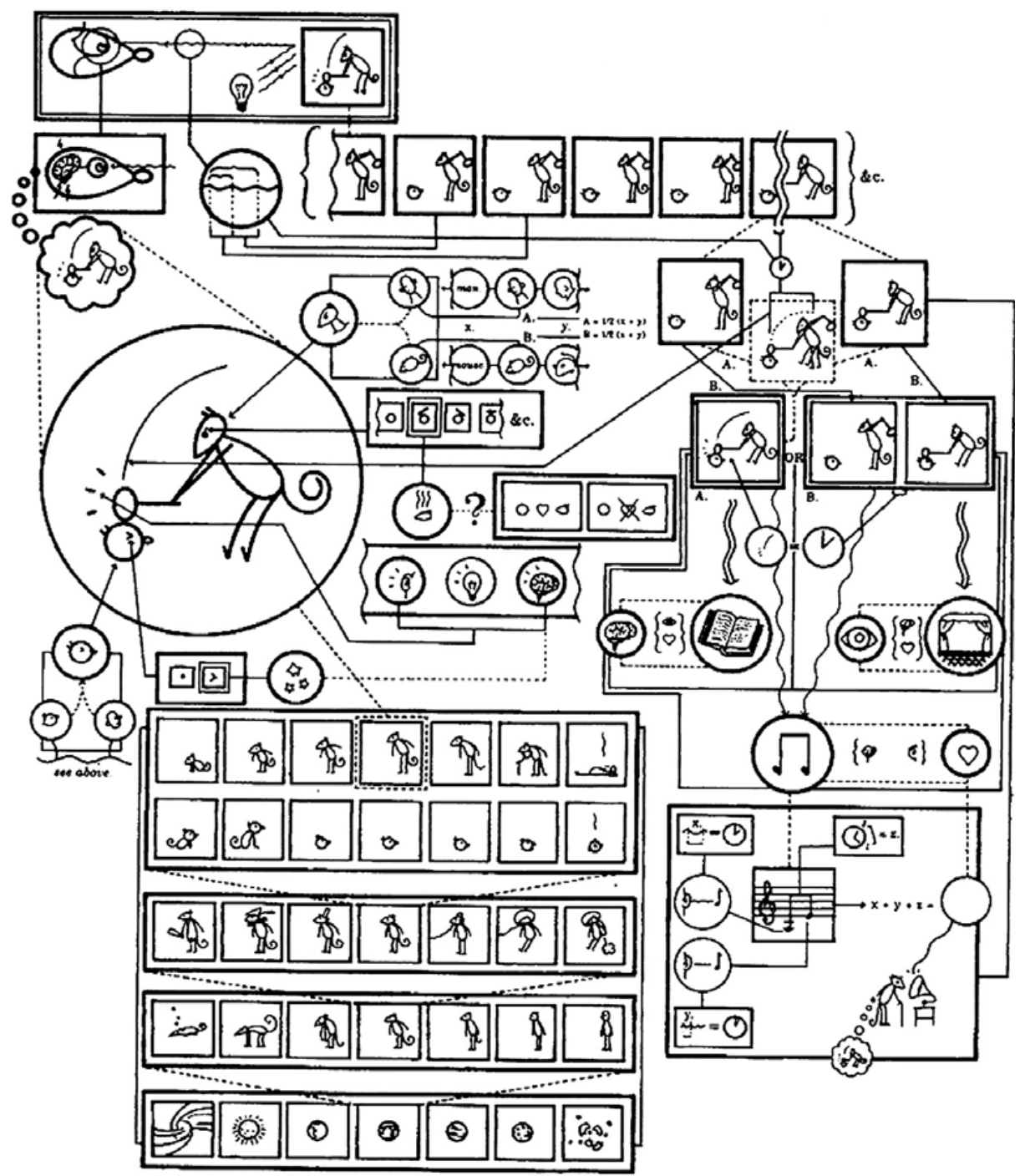

25. RAEBURN, 2004, p. 24. Le schéma (diagramme?) de Ware est commenté sous le titre: « Masturbation intellectuelle à vous fendre le crâne. La musique des bandes dessinées, illustrée » (The Imp, vol. 1, $\mathrm{n}^{\circ} 3$, p. 11). La version française est diffusée par les éditions Humeurs. 
Il existe une évidente parenté entre le diagramme où s'est condensé le fragment autobiographique de Jean Queval, et l'histoire (douloureuse) contée - ou plutôt dessinée - par Chris Ware (il faudrait lire aussi le commentaire de Daniel Raeburn). Dans les deux cas, les flèches sont fortement polysémiques. Causalité et temporalité sont à l'œuvre, en compétition. Mais d'autres liens non orientés apparaissent aussi, qui jouent un simple rôle de connexion: dans les deux cas, on est à la recherche d'une représentation graphique des composants de la signification et on cherche à exprimer, graphiquement aussi, leur articulation.

\section{LES SENTENCES ET LEUR EXÉCUTION}

Schémas, diagrammes, formes nouvelles de l'expression linguistique, phrases et « sentences » symboliques, les progrès de l'analyse ont confronté scientifiques et techniciens à des tâches d'une très grande complexité. Et c'est au début des années 1960, dans l'euphorie suscitée par les débuts de l'intelligence artificielle et les projets de documentation et de traduction automatique, que linguistes, documentalistes, spécialistes de l'analyse littéraire se sont mis à la recherche d'algorithmes et de leur implémentation informatique pour effectuer des constructions aussi complexes que celles qui viennent d'être présentées, au service de la littérature comparée, de la stylistique et même de la conception de logiciels de création littéraire assistée par ordinateur (littéraciels) ${ }^{26}$.

Si les progrès dans l'analyse syntaxique et son automatisation furent assez rapides, il n'en fut pas de même de l'analyse sémantique, face aux problèmes de la polysémie, aux pièges de la rhétorique, etc. S'il existe aujourd'hui quelques logiciels efficaces dans le domaine de la traduction automatique, surtout lorsqu'on s'intéresse à des domaines techniques spécialisés, il n'en va pas de même de l'analyse des contenus et des corrélations à longue distance. Si certains de ces espoirs ont ainsi été déçus, il n'en reste pas moins qu'un pas important a été franchi à cette occasion, un pas qui possède sans doute une grande portée épistémologique et institutionnelle: celui de la prise de conscience d'une problématique de l'automatisation au service de la maîtrise du langage naturel, automatisation rendue nécessaire par la complexité des tâches à accomplir, mais rendue possible, en même temps, par le développement rapide des techniques de calcul électronique automatique, ce qu'on appellera bientôt l'informatique ${ }^{27}$.

Ce développement s'accompagne alors de l'apparition d'une forme nouvelle de l'expression linguistique et symbolique: cette remarquable extension du domaine $\mathrm{du}$ langage que constituent les langages de programmation. Les principes de base de la programmation, telle que nous la concevons aujourd'hui, ont été posés dans un article célèbre ${ }^{28}$ où le mot « langage » n'est d'ailleurs pas prononcé. Les programmes qui déterminent le fonctionnement des machines à calculer n'en sont pas moins des textes qui,

26. C'est la tâche que s'est notamment assignée l'Atelier de littérature assistée par la mathématique et les ordinateurs (ALAMOI), groupe issu de l'Ouvroir de littérature potentielle (OULIPO), fondé en 1960 par François Le Lionnais et Raymond Queneau.

27. Un mot à succès, proposé par Philippe Dreyfus, au cours d'une conversation avec Jacques Lesourne et Jeanne Poyen.

28. Burks, Goldstine et Neumann, 1946. 
au début - et pour des raisons techniques évidentes - n'utilisent qu'un alphabet réduit (et même initialement réduit aux majuscules). Par la suite ces « langages » vont s'enrichir d'alphabets étendus, de lexiques de mots-clés réservés, comme dans FORTRAN, ou au contraire de symboles spécifiques conçus dans l'esprit du symbolisme mathématique, comme dans APL. Destinés à une forme particulière de l'expression, celle des algorithmes, ils s'inscrivent cependant, comme les langages «naturels », dans le fameux triptyque syntaxe/sémantique/pragmatique défini par Morris. Les grammaires formelles développées par Chomsky et Schützenberger n'ont d'ailleurs connu un succès durable que pour les langages de programmation et le volet sémantique peine encore à s'affirmer.

Par contre le volet pragmatique prend, avec l'informatique, une importance qui n'avait pas été soulignée par les linguistes traditionnels (à l'exception notable d'Austin, analyste précis des expressions performatives dans le langage naturel). Car dans les textes plus ou moins formalisés qu'utilise l'informatique, les entités qui apparaissent à différents niveaux - instruction, « ligne », « routine », « sous-programme », «logiciel», etc., mots, phrases ou sens - toutes s'adressent à un organe d'exécution, un processeur (soit directement via un interpréteur, soit après une phase préalable de compilation).

L'idée de « machine » est en germe, bien entendu, dans le modèle imaginé par Alan Turing $^{29}$ (et peut-être anticipé par Emil Post dès 1921) où apparaissent, dès 1936, la mémoire linéaire du ruban, le processeur (qui imprime un 0 ou un 1 sur le ruban) et surtout le mécanisme de déroulement qui permet d'effectuer les instructions d'un programme.

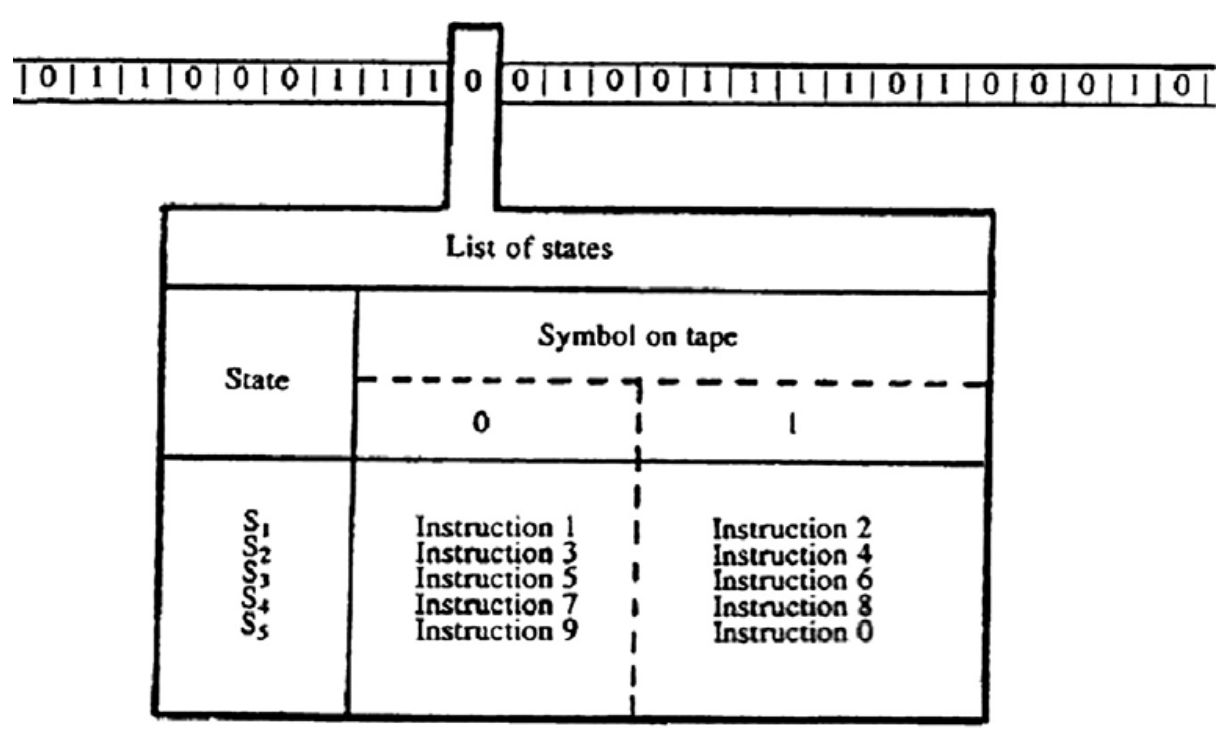

29. TURING, 1936. 
Dans la machine, le « ruban » permet l'inscription d'un texte réduit à des symboles booléens (mais il pourrait s'agir aussi bien de « lettres »); il se déplace devant une « tête de lecture ». Il est clair, ici, que les phrases - c'est-à-dire les sentences - ne doivent pas vraiment être prononcées, mais bien exécutées... Elles racontent une histoire qui, pour nous, se déroule en suivant la flèche du temps.

C'est donc un nouveau type d'événements qui apparaît ainsi. Dans les diagrammes de Feynman comme dans ceux de Grothendieck, d'autres événements étaient évoqués: interactions entre particules élémentaires, application ou projection d'un objet (ensemble ou collection) sur un autre, etc. Mais il ne s'agissait là que d'événements virtuels, ne pouvant s'actualiser que dans l'esprit du lecteur. Il n'en va évidemment pas de même en informatique où l'expression à exécuter (à interpréter, donc) - où la suite enchaînée incluant d'éventuelles boucles - déclenche effectivement une action qui modifie l'état de composants physiques (les registres) du calculateur.

Une cinématique d'un genre particulier est donc ici à l'œuvre, qui commande des actions spécifiques et en assure l'articulation. Une " phénoménologie » informatique discrète peut alors être invoquée, à l'intersection des domaines de la technologie, de la linguistique - et de l'épistémologie, là, précisément, où il est question d'ordre et d'exécution (donc de flèches...). Car certains langages de programmation comportent une opération (ou fonction) "primitive» particulière qui permet d'expliciter cette spécificité dynamique de la programmation (l'interprétation ou exécution) et d'en examiner certaines conséquences: c'est ainsi que dans le langage de programmation $\mathrm{APL}^{30}$, la primitive $\phi$ (on prononce execute, mais une première version avait été baptisée dequote) prend pour «argument» une chaîne de caractères et fournit comme « résultat» le produit du traitement de cette chaîne par l'interpréteur. C'est ainsi que le résultat de $\phi$ ' $3+4$ ' est 7 . Mais si l'on applique cette nouvelle et puissante primitive à une chaîne de caractères comprenant ce caractère $\phi$, lui-même, on peut déclencher, récursivement, des exécutions ininterrompues et engendrer ainsi la suite infinie des entiers. Cette primitive essentiellement pragmatique a en particulier le mérite de permettre l'élimination du fameux « GO TO » de la programmation traditionnelle. Elle possède aussi d'autres vertus en théorie de la complexité et de la classification des fonctions récursives ${ }^{31}$.

Plusieurs autres primitives propres à l'informatique imposaient déjà une appréhension opérationnelle du mouvement (ou des mouvements) associé(s) au fonctionnement du système et à la réalisation effective des programmes. C'est le cas de:

- l'affectation $\leftarrow:$ l'expression « $\mathrm{A} \leftarrow 3$ » entraine l'affectation de la valeur 3 à la variable A;

- la rupture de séquence $\rightarrow$ : cet équivalent du fameux « GO TO » modifie le cours d'exécution des instructions dans un programme.

Dans les deux cas l'interprétation du syntagme (plus précisément l'exécution de la sentence tout entière qui le contient) constitue un processus, déclenche une dynamique

30. Acronyme pour A Programming Language. Ce langage fut imaginé par Kenneth Iverson en 1962, mais ne connut une implémentation, pour les machines IBM, qu'en 1967. Iverson reçut, pour cela, la médaille Turing, en 1979.

31. BrafFort et Feldman, 1974, p. 373. 
qui commande le fonctionnement même du système informatique (provoquant parfois, par exemple dans le cas d'une récursivité non bornée, la saturation - et finalement l'arrêt du système hôte).

L'exécution de l'instruction informatique constitue donc une actualisation effective de l'expression formelle qui n'en réalisait que l'inscription et demeurait ainsi virtuelle: ici les sentences sont effectivement exécutées, contrairement, par exemple, aux applications, morphismes et autres « jections » de l'algèbre traditionnelle ou de la théorie des catégories.

Mais avec ces nouvelles techniques de programmation (qui utilisent ce que l'on a appelé langages «non procéduraux » que l'on oppose - contresens historique et logique - aux « langages de von Neumann »), les informaticiens ont été amenés à introduire de nouveaux concepts de base. Ainsi la «programmation objet», aujourd'hui largement répandue, utilise en particulier un concept, celui de « classe » qui n'est pas sans analogie avec le concept mathématique de catégorie. Parmi les concepts de ce genre récemment introduits, on ne s'étonnera pas de rencontrer une classe, baptisée précisément flèche qui, tout naturellement, évoque - ou voudrait évoquer-le processus élémentaire du calcul. Voici comment Ross Paterson, sur Haskell.org ${ }^{32}$, définit cette « classe » :

Arrows are a new abstract view of computation, defined by John Hughes ${ }^{33}$. They serve much the same purpose as monads - providing a common structure for libraries - but are more general. In particular they allow notions of computation that may be partially static (independent of the input) or may take multiple inputs. If your application works fine with monads, you might as well stick with them. But if you're using a structure that's very like a monad, but isn't one, maybe it's an arrow.

\section{The Arrow class}

A computation takes inputs of some type and produces outputs of another type.

A

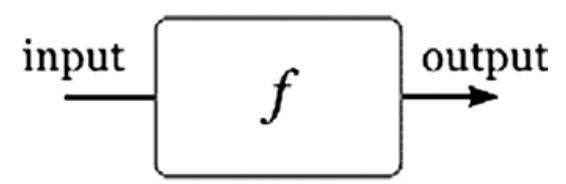

32. Voir http://www.haskell.org. Ces développements s'inscrivent en effet dans le projet de langage de programmation baptisé Haskell en hommage à Haskell B. Curry (1900-1982), initiateur de la logique combinatoire (Hilbert avait été, d'ailleurs, son directeur de thèse).

33. Hughes, 2000. 
Hence Hughes defined a Haskell class of binary type constructors:

class Arrow a where

$\operatorname{arr}::(b \rightarrow c) \rightarrow a b c$

Each function may be treated as a computation.

B

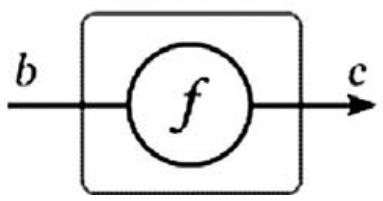

Ce sont là des notations et des schémas qui possèdent une évidente parenté avec l'univers des catégories. Mais cette parenté se manifeste ici par le biais de la programmation, et le contenu dynamique du formalisme, qui demeurait implicite dans la seule «inscription» des flèches, s'impose désormais.

La situation n'est pourtant pas aussi nouvelle qu'on pourrait le penser. Car certains mathématiciens (ou mathématiciens philosophes), probablement conscients de l'existence d'une problématique qui a évidemment sa source dans l'Antiquité même (peut-être déjà chez Archimède ${ }^{34}$ ), ont abordé - sous des angles divers - le thème de la temporalité dans les systèmes formels, et ceci bien avant qu'une « implémentation » quelconque des processus en jeu soit envisageable. On pourra ainsi citer les exemples suivants:

1) Le théoricien (mathématicien et physicien) irlandais William Rowan Hamilton (1805-1865). En plus de ses travaux essentiels en dynamique et en optique, il introduisit les concepts de vecteur et de quaternion. Mais il s'efforça, à partir de 1835, de présenter l'activité mathématique - et en premier lieu l'algèbre, comme une « science du temps pur $\gg{ }^{35}$.

2) Le mathématicien et philosophe Alfred North Whitehead (1861-1947). Auteur, avec son élève Bertrand Russell (1872-1970) des célèbres Principia mathematica (1910) qui cristallisera le mouvement « logiciste » et sera à l'origine du développement remarquable de la logique mathématique au début du $\mathrm{xx}^{\mathrm{e}}$ siècle, Whitehead deviendra le héraut de la "process philosophy », héritière d'Héraclite et de Leibniz (mais proche aussi de Bergson et de Peirce), institutionnalisée avec le Center for Process Studies et la revue éponyme ${ }^{36}$.

3) Mais avant lui, le géomètre et logicien allemand Moritz Pasch (1843-1930), connu pour son axiomatisation de la géométrie euclidienne (l'axiome de Pasch est l'un

34. La fameuse argumentation dite de «l'Arénaire », où les concepts d'échelle et de récursivité semblent apparaître, peut être citée ici.

35. Cette première publication était intitulée: Theory of Conjugate Functions, or Algebraic Couples and Elementary Essay on Algebra as the Science of Pure Time. Voir, à ce sujet, l'article très documenté de ØнRstrøм, 1985.

36. Voir en particulier l'ouvrage fondamental de WhiteHEAD, 1978. 
des fondements de la géométrie), s'efforça de développer sa vision « empiriste » sur la base de «noyaux de concepts fondamentaux » formant la partie pré-mathématique de la discipline ${ }^{37}$. Heyting, dans une analyse succincte, énumère ainsi ces concepts :

1) Chose, c'est-à-dire objet perceptible;

2) Événement, les événements sont des choses;

3) Donnée d'une chose, sorte particulière d'événement;

4) Nom propre, tous les noms sont des choses;

5) Nom collectif, c'est-à-dire nom générique pour des choses données;

6) Événement antérieur ou postérieur;

7) Événement immédiatement consécutif;

8) Chaîne d'événements, chose qui résume un nombre quelconque d'événements vécus.

Pasch est le premier (et fut longtemps le seul, avec les considérations inabouties de Brouwer sur les « arguments historiques ») à préconiser une « cinématique abstraite » de ce genre.

Mais ce sont les recherches sur le fondement des mathématiques et, plus précisément, sur la non-contradiction de l'arithmétique qui, au début des années 1930, donneront un sens plus concret à cette notion de processus. Certes les « machines de Turing » ne sont pas de véritables machines et n'ont pas inspiré la conception des premiers ordinateurs (auxquels Turing travaillera après la guerre). Il existe d'ailleurs un certain malentendu relatif au rôle décisif de John von Neumann. Car, indépendamment de la conception des premières calculatrices électroniques (destinées, à l'origine, à la résolution des systèmes d'équations aux dérivées partielles - celles de l'hydrodynamique en particulier), von Neumann, inspiré par les travaux de McCulloch et Pitts ${ }^{38}$, propose aussi une Théorie Générale et logique des automates ${ }^{39}$ qui introduit pour la première fois la notion d'automate cellulaire. Cette notion qui est explicitement, comme pour McCulloch et Pitts, une modélisation des réseaux de neurones, va inspirer de nombreux chercheurs, mathématiciens plutôt qu'ingénieurs.

C'est en 1970 que Martin Gardner décrivit l'invention de John Conway (mathématicien anglais, spécialiste éminent de la théorie des groupes finis): le « Jeu de la Vie (Game of Life) $»^{40}$. Il s'agit d'un modèle formel d'automate, défini par des règles très simples qui déterminent le comportement d'une configuration d'entités binaires (cellules « vivantes » ou « mortes ») sur un ensemble potentiellement infini de cases. Le jeu de règles fut déterminé par Conway, après un certain nombre d'essais qu'il effectuait en utilisant un « ban » de go comme grille, espace de développement pour les organismes élémentaires et les pierres du jeu de go pour matérialiser les cellules de ces

37. PASCH, 1920-1921 et 1927.

38. McCulloch et Pitts, 1943.

39. Neumann, 1996 (la version originale a été publiée par John Wiley en 1951).

40. «Mathematical Games. The Fantastic Combinations of John Conway's New Solitaire Game "Life" », Scientific America, n 223, oct. 1970, p. 120-123. 
organismes. Voici une présentation imagée des règles de ce jeu: tout d'abord le « flipflop ». Les deux configurations suivantes sont « stables »:

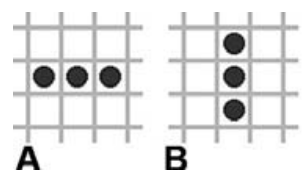

Si une cellule a exactement trois voisines vivantes, elle demeure vivante:

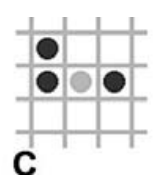

Si une cellule a exactement deux voisines, elle reste dans son état actuel à l'étape suivante. Si une cellule a strictement moins de deux ou strictement plus de trois voisines vivantes, elle est morte à l'étape suivante. C'est le cas de la cellule noire dans la configuration ci-dessous :

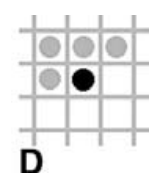

Le jeu se développa rapidement dans les universités américaines et reçut des implémentations informatiques permettant de réaliser d'innombrables simulations qui mirent en valeur des configurations intéressantes: «bloc », " grenouille», " glider » comme ci-dessous (mais il faut évidemment disposer d'un lien avec le Web pour que le « glissement » apparaisse et se propage sous nos yeux). En voici un «snapshot»:

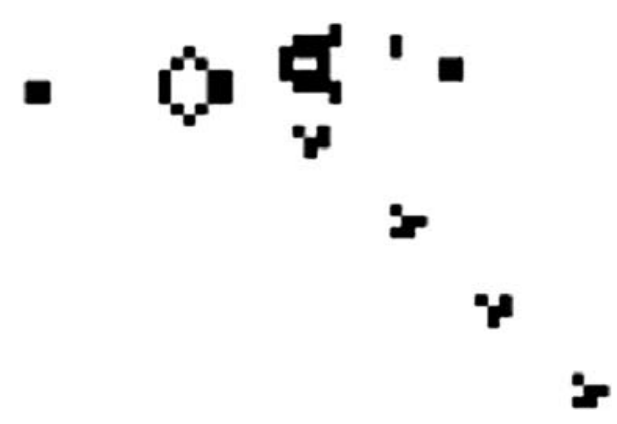

Les « automates de Conway » donnèrent lieu à de nombreuses analyses théoriques et logiques. Moyennant un codage adéquat des « configurations » initiales, on a montré qu'il était possible de simuler le comportement de « machines de Turing », et même de machines de Turing universelles - donc de tout algorithme effectif.

L'étude des automates cellulaires connut un développement encore plus spectaculaire avec le projet gigantesque de Stephen Wolfram. Mathématicien et théoricien 
précoce, celui-ci abandonna la physique théorique pour développer un outil de calcul formel assisté par ordinateur: Mathematica. Séduit par la richesse et la variété des «organismes» engendrés, Wolfram en vint à concevoir une nouvelle forme d'universalité. De 1982 à 2002, il imagina un très grand nombre de modèles d'automates cellulaires associés à des règles d'engendrement variées. La règle 110, illustrée ci-dessous, lui parut exemplaire (ici aussi il est recommandé d'observer le développement du processus sur un écran d'ordinateur):

Rule 110

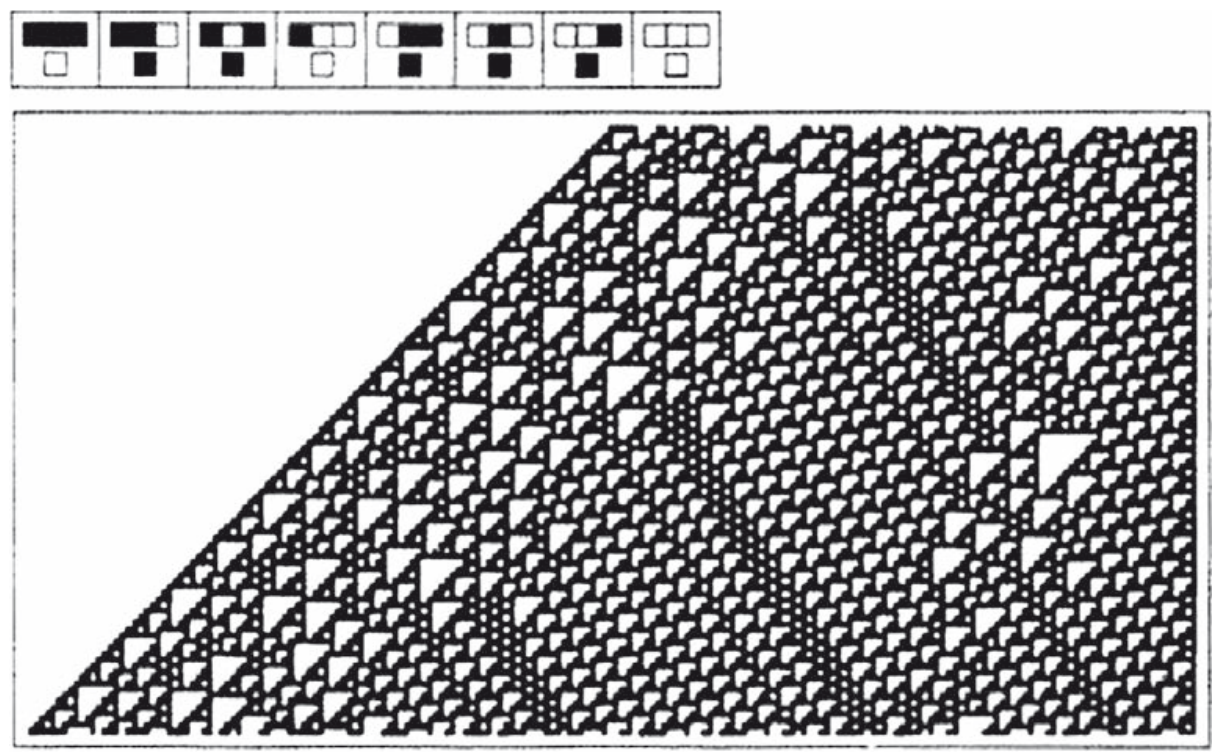

Fragment d'image engendré par la règle 110.

Ce caractère d' " universalité » amena Wolfram à imaginer un système complet des sciences: physique, biologie, psychologie, sociologie, etc., qui abandonnerait le formalisme mathématique (formalisme qu'il maîtrise pourtant parfaitement) en faveur de modèles d'automates cellulaires ${ }^{41}$ associés à des configurations initiales convenablement choisies. Les " organismes » ou « configurations » remplaceraient alors les équations et autres structures mathématiques. Il développa de nombreuses applications de ses principes, en cryptographie, en hydrodynamique et il prétendit avoir défini une machine de Turing universelle ne possédant que deux « états » et deux « couleurs ».

Il se développe ainsi, depuis une quinzaine d'années, un mouvement d'enthousiasme technologique qui remonte en particulier à Edward Fredkin et même, prétendent certains, à Leibniz (mais, de façon plus directe, à John von Neumann et à John Conway) autour d'un concept très général de machine et, plus particulièrement, de machine digitale: une véritable « philosophie digitale ». Fredkin, né en 1934, est concepteur de systèmes

41. Voir A New Kind of Science, publié en 1982 par la société Wolfram Research. 
et de procédures informatiques; il est l'inventeur, en particulier, des « Fredkin gates ». Dans une série de publications - Digital machines (1990), A New Cosmogony (1992), Finite Nature (1992), On the Soul (2000), Introduction to Digital Philosophy (2006) Fredkin développe un modèle d'univers entièrement fini (et même « discret ») fonctionnant comme un immense «Two-State, Reversible, Universal Cellular Automata in Three Dimensions $»^{42}$. Les événements d'un tel univers ne sont alors que la manifestation de programmes exécutés par les automates qui le constituent.

Encore plus enthousiaste, Ray Kurzweil (né en 1948), héraut de l'intelligence artificielle et inventeur célèbre de nombreux et importants outils: reconnaissance de caractères écrits et traduction de la chaîne écrite en chaîne parlée (à l'intention des aveugles), construction d'instruments de musique "parfaits », génération de textes littéraires assistée par ordinateur, etc., se lança progressivement dans une entreprise encore plus audacieuse que celle de Wolfram (dont il a commenté par ailleurs le travail avec sympathie, mais aussi quelques réserves). Après des ouvrages de plus en plus ambitieux $^{43}$, il a publié récemment une sorte de « pronostication » des plus optimistes, décrivant les progrès technologiques les plus spectaculaires (y compris l'accès à l'immortalité dès 2045 lorsque apparaît la « singularité »... $)^{44}$.

À l'issue des « six époques » qu'il distingue dans l'évolution de l'univers - «Physics and Chemistry», "Biology and DNA», «Brains », "Technology», "The Merger of Human Technology with Human Intelligence », «The Universe Wakes Up» - arrive le moment proche (2042, "The Singularity is Near »), où tout est devenu possible: immortalité, maîtrise du cosmos, etc. Kurzweil n'hésite pas à préciser les nombreuses étapes qu'il imagine le long du parcours de cette flèche du temps : 2020-2050-2070 (âge des machines intelligentes); 2019-2029-2049-2072-2099 (âge des machines spirituelles); 2045 (la « singularité »), etc. Certaines ont été atteintes, conformément aux prévisions, d'autres non. La plus impressionnante, évoquant la singularité, est encore devant nous. Mais n'oublions pas que ces anticipations ont été proposées sur la base d'un modèle totalement digital de l'univers.

\section{CIBLES ANCIENNES ET NOUVELLES}

\section{Cibles anciennes}

Dans les premiers moments du développement des techniques de calcul automatique, méthodes et techniques se sont longtemps partagées en deux classes: celle du digital et celle de l'analogique. Il est vrai que les progrès de l'électronique ont engendré la disparition presque complète de la seconde classe en ce qui concerne, du moins, le calcul automatique. Mais le thème de l'analogie, qui remonte à Aristote et aux Pères de l'Église, n'a cessé d'être présent à l'esprit des chercheurs et des épistémologues, sous des formes diverses.

42. Le texte intégral est accessible à l'adresse suivante: http://arxiv.org/ftp/nlin/papers/0501/ 0501022.pdf.

43. KurzweIL, 1990 et 1999.

44. KurZweIL, 2005. 
C'est Norbert Wiener (il avait débuté comme logicien, avec Bertrand Russell) qui donnera (après Vannevar Bush et sans connaître l'œuvre de Michel Petrovitch), un nouveau départ à un mouvement « analogiste», purement scientifique. Étudiant les processus stochastiques, il est conduit, après un séjour à l'Institut de cardiologie de Mexico, en 1944, à entreprendre, avec Arturo Rosenblueth, une collaboration qui va le conduire à imaginer une discipline, la cybernétique qui va rapidement connaître un essor considérable. Aux confins de la neurophysiologie, de l'ingénierie (et en particulier de l'automatique), cette discipline nouvelle (dont le nom avait déjà été proposé par Ampère) met en œuvre de nouvelles structures de diagrammes et schémas permettant d'interpréter la dynamique de certains processus et en proposer éventuellement une modélisation mathématique ${ }^{45}$.

Les applications de la cybernétique sont nombreuses et précoces. Dès 1956, un Congrès international est organisé à Namur. Le Congrès, présidé par François Le Lionnais (en remplacement de Pierre Auger), a comporté les sections suivantes: 1) Principes et méthodes de la cybernétique; 2) Les machines sémantiques ; 3) L'automation; 4) La cybernétique et la vie. De nombreux autres congrès et colloques suivront.

La cybernétique imagine et construit des machines où s'inscrivaient des diagrammes et des flèches dont la signification est bien particulière: il s'agit ici, d'un autre type bien particulier de mouvement, celui de l'information transportée sur la ligne qu'elles accompagnent et orientent. Et lorsque ces chemins de l'information comportent une boucle, on se trouve dans une situation originale dont Wiener a, le premier, souligné l'importance ${ }^{46}$ : c'est la boucle de feedback qui devient le maître-mot de la cybernétique (traduit par Pierre de Latil par « rétroaction $»^{47}$ ). La flèche, ici, n'indique pas seulement une orientation: elle explicite une topologie dont le rôle est essentiel.

Dans l'ouvrage de Wiener, comme dans celui de Latil, d'importants aspects de ces boucles sur les plans mathématique et logique sont décrits et analysés. Dans le chapitre IV, Feed-back and oscillations, est introduit le concept de «control flow chart» (diagramme de contrôle du flux) ${ }^{48}$ qui deviendra familier aux spécialistes du calcul analogique. En voici un exemple où, sans doute pour la première fois, apparaît, dans un diagramme, une boucle où l'information est réinjectée, avec l'information initiale, sur l'entrée du processeur électronique où se développent les calculs. C'est le premier exemple de rétroaction (voir le schéma de Wiener, en haut de la page 93).

Wiener développe alors l'analyse qui permet d'obtenir une description mathématique du processus et d'en prévoir le résultat. Une version simplifiée de ce schéma permet d'aller plus loin dans l'analyse qui illustre ce que l'on peut appeler le paradoxe des unités opérationnelles dans le calcul analogique. C'est ce paradoxe qu'illustre le schéma situé en dessous du schéma de Wiener.

45. On lira avec plaisir les essais autobiographiques de Norbert Wiener, en particulier WIENER, 1956 (vol. II); voir aussi Triclot, 2008.

46. Le livre fondateur de Norbert Wiener était sous-titré Control and Communication in the Animal and the Machine. Voir WIENER, 1948.

47. LATIL, 1953.

48. WIENER, 1948, p. 112. 

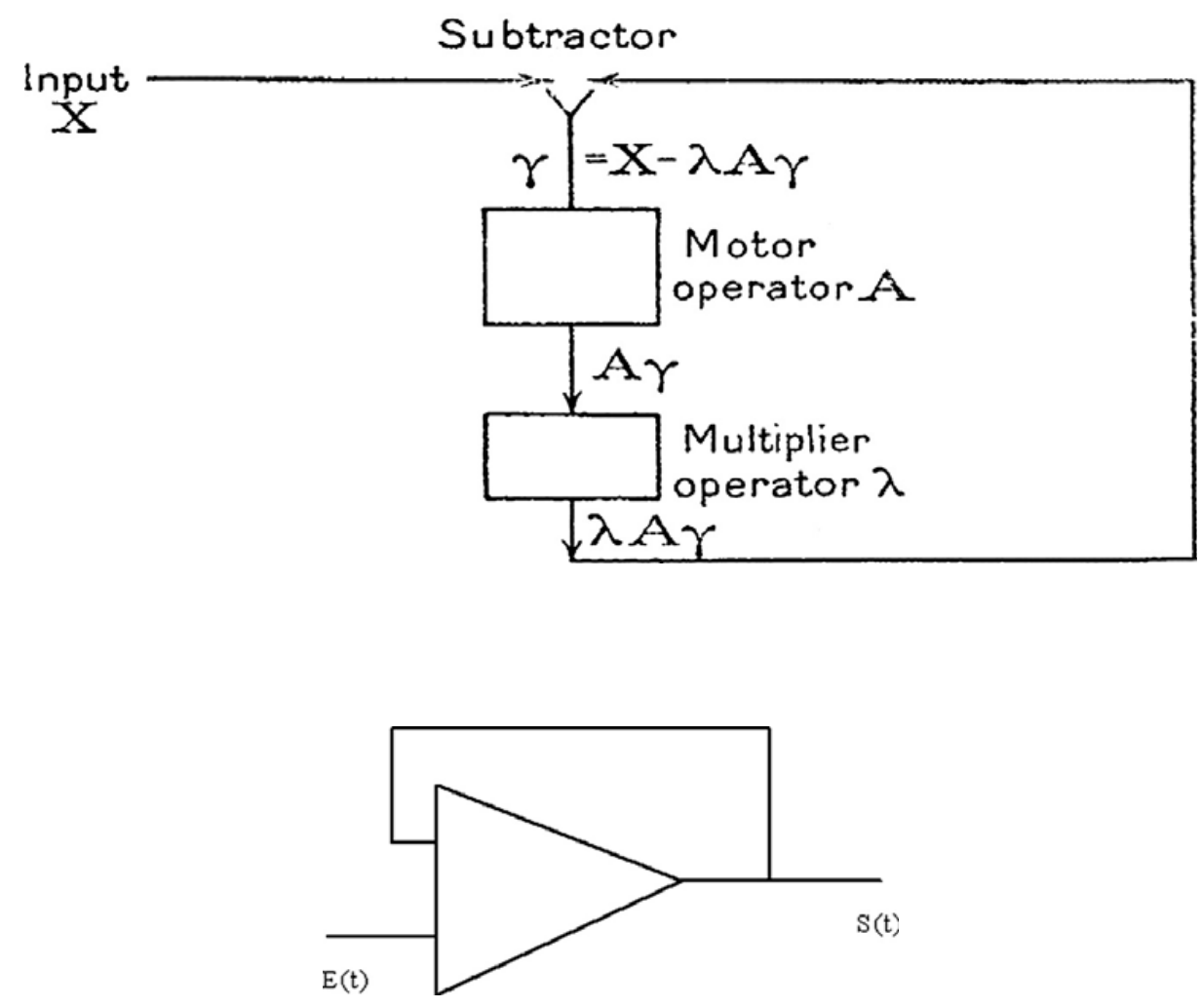

Il semble intéressant d'en approfondir l'analyse qui peut s'expliciter ainsi :

1) L'objectif recherché, dans la constitution de ce schéma analogique, est d'obtenir la valeur opposée d'une valeur donnée (représentée par une tension qui varie dans le temps): $\mathrm{S}(\mathrm{t})=-\mathrm{E}(\mathrm{t})$.

2) En raison du bouclage de la sortie sur l'entrée, la tension électrique à l'entrée, $E(t)$, et la tension de sortie $S(t)$ s'ajoutent algébriquement.

3) Soit alors $\varepsilon(\mathrm{t})=\mathrm{E}(\mathrm{t})+\mathrm{S}(\mathrm{t})$ : c'est la grandeur qui se présente à l'entrée de l'amplificateur (que l'on qualifie souvent d'amplificateur fonctionnel). Cette valeur $\varepsilon(\mathrm{t})$ est alors multipliée par le gain $\mathrm{G}$ de l'amplificateur.

4) Comme ce gain est très grand (de l'ordre de $\left.10^{5}\right), \varepsilon(\mathrm{t})$ doit être très petit pour que la sortie, $\mathrm{S}(\mathrm{t})$, qui est donc égale à $\mathrm{G} \times \varepsilon(\mathrm{t})$, soit finie. C'est la boucle de rétroaction qui permet d'obtenir ce résultat.

5) Dans ces conditions, $\mathrm{S}(\mathrm{t})$ est très voisin de $-\mathrm{E}(\mathrm{t})$, ce qui est le résultat désiré. Très voisin, mais pas identique : $\varepsilon(\mathrm{t})$ est bien l'erreur commise dans ce calcul et la solution, $\mathrm{S}(\mathrm{t})$, n'existe donc que parce qu'il subsiste une erreur!

C'est là le paradoxe du calcul analogique, paradoxe qui commande le fonctionnement de tous les schémas: la rétroaction fonde la solution. 
Ce paradoxe n'est évidemment pas de la même nature que ceux qui étaient apparus lors de l'approfondissement de la théorie des ensembles, au début du $\mathrm{xx}^{\mathrm{e}}$ siècle (mais qui, pour l'essentiel, étaient connus dès l'Antiquité) : paradoxes logiques ou sémantiques où des boucles, souvent, sont à l'œuvre, en effet, mais dont la dynamique demeure implicite. Ce sont eux, cependant, qui ont attiré l'attention des théoriciens du calcul électronique plus familiers, sans doute, avec la logique traditionnelle (booléenne) qu'utilisent les constituants de base du calcul digital.

Le calcul analogique, par contre, s'est appuyé sur des éléments de théorisation issus de l'électronique. La notion, familière à l'électronicien, de «block-diagram » a donné naissance, avec Mason, au concept de «signal flow diagrams », dont il a proposé une théorie ${ }^{49}$.

Peu après, ce concept trouva une application naturelle dans la formalisation des schémas du calcul analogique, une forme de mathématique appliquée devenue essentielle pour la simulation de structures technologiques complexes: aérodynamique, ingénierie nucléaire, industrie spatiale, etc.

Les « diagrammes de fluence » explicitent la structure d'un système d'équations algébriques (les arcs n'y sont pas orientés). Les schémas analogiques, par contre, qui les complètent et s'y combinent, permettent d'y inclure des systèmes d'équations différentielles et d'introduire pour cela des relations de dépendance supplémentaires (certains arcs doivent ainsi être orientés). Ce sont alors des diagrammes informationnels où l'on est amené à définir des conditions de compatibilité relatives au flux des informations tant algébriques qu'analytiques. Ces conditions permettent alors de procéder à l'orientation des arcs demeurés non orienté ${ }^{50}$.

Cette «complétion sagittale » combine considérations logiques, algébriques et analytiques au sein d'une méthodologie originale qui n'a sans doute pas été complètement exploitée, le calcul analogique ne s'étant plus développé, faute notamment d'enjeux industriels. Il semble pourtant qu'elle pourrait trouver sa place, dans le cadre d'une épistémologie sagittale.

L'exemple, page suivante, illustre cette problématique où s'inscrivent, dans le cadre de la simulation d'un grand système technologique, l'argumentation topologique en question et, implicitement, le paradoxe du calcul analogique.

Le schéma présenté prépare, avec son diagramme informationnel, la simulation d'une dynamique: celle d'un système couplé « réacteur nucléaire/échangeur de température » (on y calcule l'évolution des variables essentielles: températures des fluides, pressions, etc.). La prise en compte des contraintes de ce qu'on pourrait appeler la « complétion sagittale » permet de distinguer - sans calcul explicite - une configuration « diagrammatiquement» possible d'une configuration impossible ${ }^{51}$.

Malgré le succès des simulations analogiques en physique appliquée et en ingénierie (diffusion hydrodynamiques ou thermodynamiques, calcul de trajectoires, prévisions météorologiques, etc.), les ingénieurs ont abandonné, depuis quelques

49. MASON, 1953 et 1956.

50. BrafFort et Caillet, 1957.

51. Voir les détails dans Braffort et Caillet, 1959, p.15. 


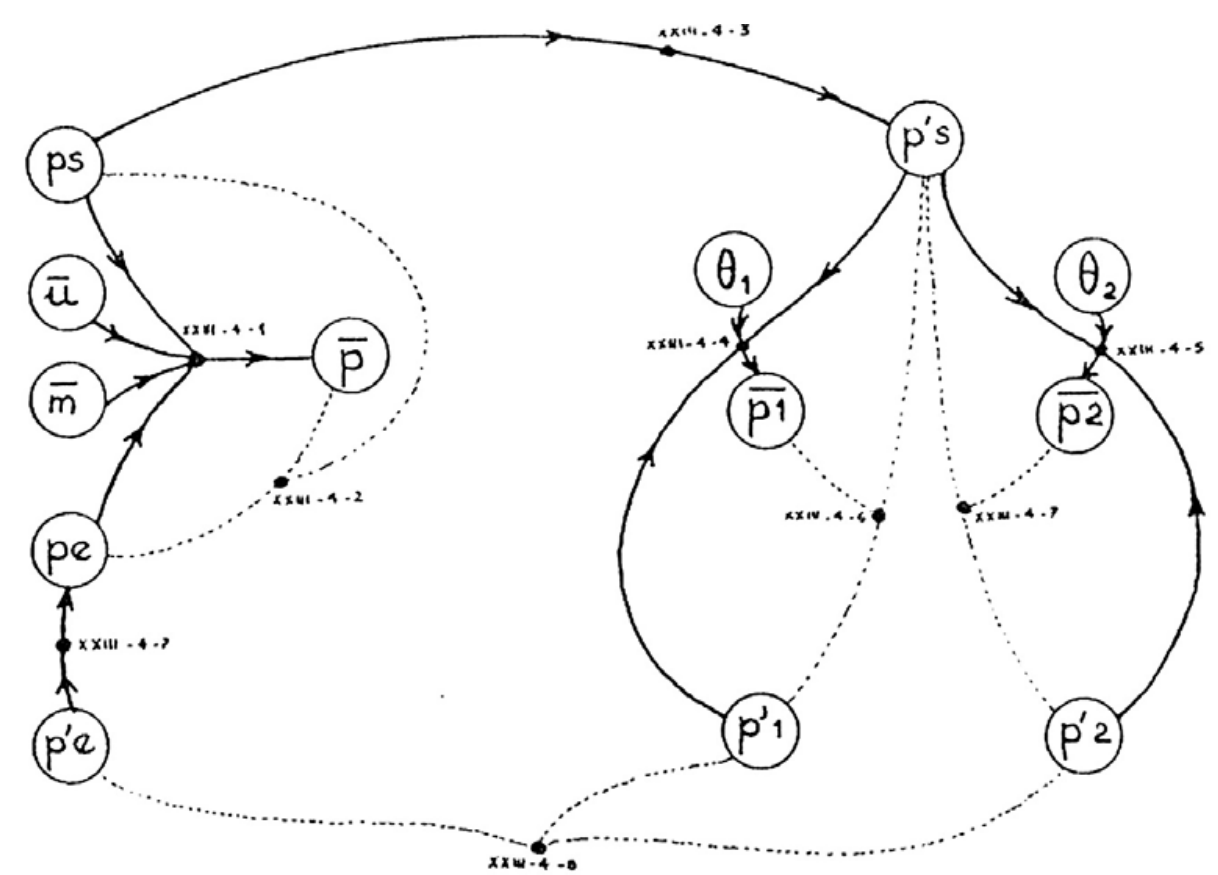

Schéma mathématique, ou partiellement ordonné, du système $A$
5 équations différentielles
4. relations algébriques
9 fonctions inconnues.

Les numéros XXIII-4-... sont ceux des équations.

Les lettres encerclées sont les indices correspondant aux différentes températures inconnues

Diagramme informationnel associé à un système d'équations algébriques et différentielles

décennies, les techniques du calcul analogique en faveur des techniques du calcul digital, que les progrès technologiques ont rendu infiniment plus précises sans que la vitesse d'exécution des calculs soit encore rédhibitoire, comme elle le fut longtemps ${ }^{52}$.

La « philosophie digitale » l'emporte donc. Mais n'est-ce pas là une nouvelle manifestation de cette regrettable "amnésie » que Jean-Marc Lévy-Leblond a souvent dénoncée chez les scientifiques? (Les philosophes n'en étant pas immunisés, bien entendu !) Un rappel y portera peut-être remède.

Mère de la cybernétique, la pensée analogique est également fille de la phénoménologie et s'est même constituée en discipline distincte dès le XVIII ${ }^{e}$ siècle. Son véritable initiateur est Johann Heinrich Lambert (1728-1777). Mais c'est Michel Petrovitch qui donna son véritable essor à une phénoménologie scientifique. Au début de son

52. Voir, par exemple, FARGE, 2007. 
intervention à la séance d'ouverture du premier congrès international de cybernétique (Namur, 1956), le philosophe Léon Delpech rappelait:

«En 1907, Gauthier-Villars publiait un ouvrage du philosophe yougoslave Petrovitch intitulé La Mécanique des phénomènes fondés sur les analogies. Il s'agissait pour son auteur de montrer que certaines particularités de l'allure d'un phénomène, peuvent s'expliquer par des mécanismes communs à un grand nombre de phénomènes divers et que ces mécanismes sont fournis par des schémas généraux. Première tentative de mécanique comparée que Petrovitch devait compléter en 1921 par la parution chez Alcan de ses Mécanismes communs aux phénomènes disparates ${ }^{53}$. »

C'est dans une lettre à Georges Sagnac, au début de 1898, que Michel Petrovitch évoque son projet d'une nouvelle science, une science unitaire qu'il baptisera « phénoménologie ». Ses deux premières communications dans ce domaine sont publiées en serbe: Aperçu sur la géométrie des masses (1896) et Théorie mathématique de l'activité des causes (1899), immédiatement suivis par un texte en français: Les Analogies mathématiques et la Philosophie naturelle (1901). Dès février 1906, les éditions GauthierVillars publient La Mécanique des phénomènes fondée sur les analogies. Et c'est en 1921 que Mécanismes communs aux phénomènes disparates paraît chez Félix Alcan $^{54}$. Il faut souligner que la "phénoménologie générale », n'est pas seulement une construction formelle. Elle débouche sur des expériences et sur la construction d'équipements sophistiqués, notamment de machines analogiques. Les flèches, bien entendu, y abondent:

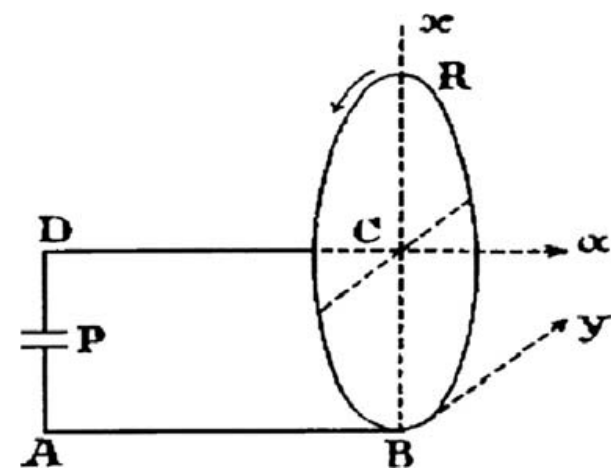

\section{Cibles nouvelles}

Les schémas de l'ingénierie, comme les diagrammes analogiques ainsi qu'heuristiques, de l'algèbre catégorique et d'autres domaines scientifiques et techniques, donnent toute leur importance aux flèches, dans leurs divers emplois: logique, dynamique, eidétiques. Aussi leur absence presque totale du débat épistémologique n'est-elle

53. Voir les Actes du Ir Congrès international de cybernétique, parus chez Gauthier-Villars, en 1958 (p. 120-121).

54. Pour plus de détails sur Lambert et Petrovitch, voir BRAFFORT, 2007-2008. 
sans doute que provisoire. Mais le retour qui, me semble-t-il, se profile, ne se fera pas uniquement à l'aide de développements théoriques, de textes ou de formules (et de diagrammes), mais plutôt de machines, et précisément de machines réelles, fonctionnant dans le temps et dans l'espace.

Comme souvent, ce sont les artistes ou des techniciens qui montreront sans doute le chemin, un chemin où se rencontreront les nécessités de la représentation précise des événements et une expression fidèle des composantes dynamiques qui les animent.

On peut évidemment citer ici les systèmes de notations pour la chorégraphie, systèmes au très riche passé (le premier système fut publié en 1588): ils se sont beaucoup développés au Xx ${ }^{\mathrm{e}}$ siècle, avec Rudolf Laban (1928) puis Rudolf et Joan Benesh (1956). Le CIREN (Centre interdisciplinaire de recherche sur l'esthétique du numérique, Université de Paris 8), dans sa recherche sur les «nouvelles écritures scéniques », étudie « Les écritures chorégraphiques et la notation assistée par informatique ». Une exposition a été organisée au Centre national de la danse, en 2006-2007, sur Les Écritures du mouvement, au cours de laquelle Marion Bastien a présenté son logiciel LabanWriter, un éditeur de partition en notation Laban. Un exemple de cette notation figure ci-dessous.

Des études et des notations semblables ont été utilisées en ergonomie industrielle ${ }^{55}$. Il s'agit donc, ici encore, d'une notation exécutable. Réciproquement les spécialistes de la «Langue des Signes » et en particulier de la «Langue des Signes Française »,

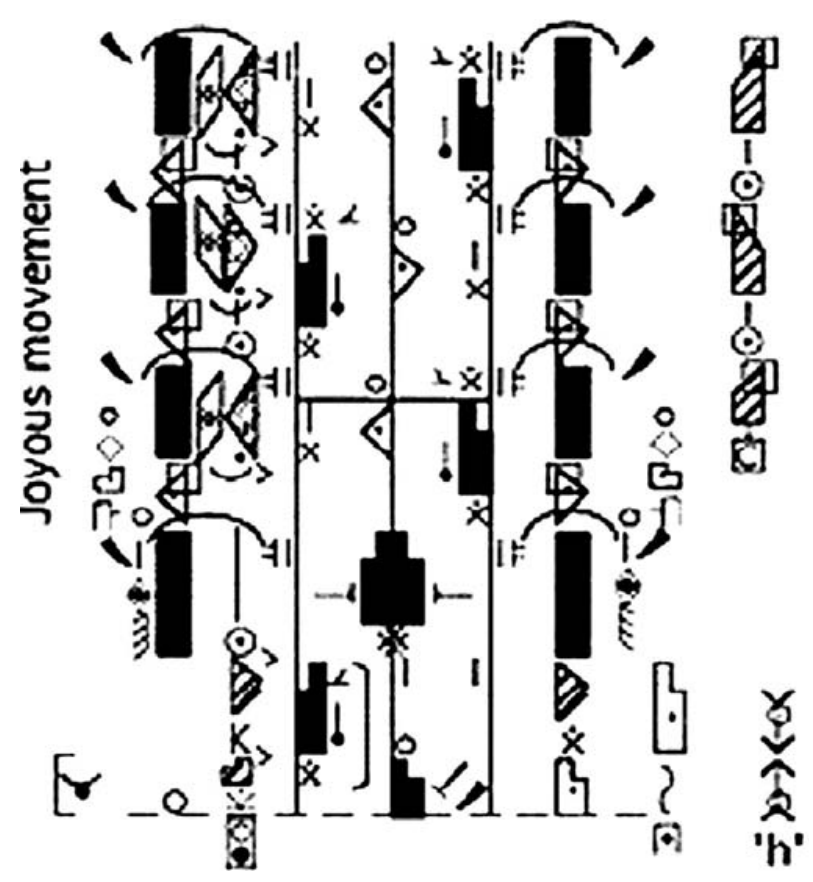

55. Preston-Dunlop, 1968. 
évidemment impliqués dans le travail sur les gestes ${ }^{56}$, s'orientent activement vers le développement d'une notation graphique spécifique ${ }^{57}$. Le rapport final du projet $L S$ Script, commun à Paris 8 , à l'Iris, à Websourd, à l'IRIT (Toulouse) et au LIMSI-CNRS (Orsay) a été présenté en juin 2007. Bien entendu, il sera question de la possibilité de la traduction automatique du langage " signé » dans le formalisme graphique ainsi défini de nouvelles «machines », une nouvelle « interaction homme-machine». En méditant ainsi sur les possibilités qu'offrent les outils informatiques pour l'expression, et l'implémentation effective d'événements dynamiques sur un écran d'ordinateur, en imaginant et en spécifiant des formalismes non parlés, ou au contraire non exprimables par des signes inscrits sur un support statique, on est conduit à réfléchir à ce que serait une science nouvelle qui exploiterait un formalisme effectivement dynamique, dans les domaines de l'analyse comme de la recherche appliquée.

Ces observations ne sont pas vraiment nouvelles. Énoncées dès le congrès de cybernétique de Namur ${ }^{58}$, elles se sont précisées peu à peu ${ }^{59}$ mais ne pouvaient s'appuyer sur des implémentations effectives que seules les nouvelles technologies pouvaient rendre possible. Elles rejoignent celles d'autres auteurs comme Jacques Mandelbrot, physicien, mais également peintre, qui évoque un souvenir de Jacques Hadamard à qui Einstein confiait que, jusqu'à un stade avancé du raisonnement, sa pensée se présentait à lui sous forme d'images et plus encore d'impulsions musculaires ${ }^{60}$.

Que serait donc, par exemple, une mathématique aux objets « vivants », aux foncteurs « actifs", retrouvant peut-être les méditations de Brouwer sur les " arguments de priorité » comme ceux de Pasch sur l'origine (Ursprung) de l'arithmétique et les projections graphiques de Chris Ware? Que serait une physique, une science où simulation serait en même temps explication (mais plus convaincante que celles de Wolfram ou de Fredkin)?

Nouveau formalisme, mais aussi nouvelles machines pour le mettre en œuvre, ce formalisme qui serait aussi un transformalisme. Ce serait sans doute le jugement des flèches qui serait ainsi prononcé.

56. BRAFFORT (Annelies), 1996.

57. LS Script. Projet RIAM 2005-2007.

58. BRAFFORT, 1956a et $1956 \mathrm{~b}$.

59. BRAFFORT, 1977 et 1989.

60. MANDELBRot, 2007. 


\section{LISTE DES RÉFÉRENCES}

Beaugrande (Robert de) et Dressler (Wolfgang), 1983, Introduction to Text Linguistics, Londres, Longman.

Braffort (Annelies), 1996, Reconnaissance et compréhension de gestes. Application à la langue des signes, thèse de doctorat de l'université de Paris XI (http://www.limsi.fr/Scientifique/ geste/Docs/TheseAnneliesBraffort.pdf).

BRAFFort (Paul), 1956a, « Cybernétique et physiologie généralisée », dans Actes du I ${ }^{e r}$ Congrès international de cybernétique, Paris, Gauthier-Villars, p.102.

BRAFFORT (P.), 1956b, « L'information dans les mathématiques pures et dans les machines », dans Actes du I ${ }^{e r}$ Congrès international de cybernétique, Paris, Gauthier-Villars, p. 248-249.

BrafFort (P.), 1968, L'Intelligence artificielle, Paris, Presses universitaires de France.

BRAFFort (P.), 1977, Formalisme, déformalisme, transformalisme. Une recherche de voies nouvelles en systématique notationnelle, Rapport interne de la société GAI (Génie automatique et informatique), Bagneux, document dactylographié.

Braffort (P.), 1989, « Le dessein des mots animés », communication au Colloque des arts électroniques, Rennes, juin (http://alamo.mshparisnord.org/publications/DesMAn.html).

BRAFFORT (P.), 1999, Science et littérature. Les deux cultures, dialogues et controverses pour l'an 2000, Paris, Diderot.

Braffort (P.), 2007-2008, «La deuxième vie de Michel Petrovitch », Epistémocritique. Revue [électronique] d'études et de recherches sur la littérature et les savoirs (http://www.epistemocritique.org/), I ${ }^{\text {er }}$ part. : 4 juin 2007; II part. : 15 janvier 2008.

Braffort (Paul) et CAILlet (Claude), 1957, «La dynamique d'un réacteur à modérateur circulant », Proceedings of the I.E.E., vol. 104, p. 437.

Braffort (P.) et Caillet (C.), 1959, « Schémas analogiques et diagrammes fonctionnels dans l'étude des problèmes de stabilité », Cours de génie atomique, vol. XXIII, Saclay, Commissariat à l'énergie atomique.

BrafFort (Paul) et Feldman (Denis), 1974, « Some Recursion-Theoretic Uses of the "Dequote" Operator », Journal of Symbolic Logic, vol. 39, p. 373.

BrafFort (Paul) et Leroy (André), 1959, «Des mots-clés aux phrases-clés. Les progrès du codage et l'automatisation des fonctions documentaires ", Bulletin des bibliothèques de France, t. IV, n 9, p. 383-391 (http://bbf.enssib.fr).

Burks (Arthur), Goldstine (Herman) et Neumann (John von), 1946, Preliminary Discussion of the Logical Design of an Electronic Computing Instrument, Princeton, Institute for Advanced Study.

Cramer (John G.), 1983, «The Arrow of Electromagnetic Time and Generalized Absorber Theory ", Foundations of Physics, vol. 13, p. 887.

Eilenberg (Samuel) et Mac Lane (Saunders), 1945, « General Theory of Natural Equivalences », Transactions of the American Mathematical Society, vol. 58, p. 231-294.

Farge (Marie), 2007, «Numerical Experimentation. A Third Way to Study Nature », dans Kaneda (Yukio), Kawamura (Hiroshi) et Sasai (Masaki), éd., Frontiers of Computational Sciences, New York, Springer, p. 15-30.

Feys (Robert) et Fitch (Frederic B.), 1969, Dictionary of Symbols of Mathematical Logic, Londres, North-Holland. 
Hughes (John), 2000, « Generalising Monads to Arrows », Science of Computer Programming, vol. 37, p. 67-111.

JEDRZEJEWSKI (Franck), 2007, Diagrammes et catégories, thèse de doctorat de l'université DenisDiderot, Paris (http://tel.archives-ouvertes.fr/tel-00193292/fr/).

Johnson (Mark), 1987, The Spiritual Logic of Ramon Llull, Oxford, Clarendon Press.

KAC (Eduardo), 1999, « Genesis », installation reproduite dans Signs of Life, Cambridge, MIT Press, 2007.

KurzweIL (Ray), 1990, The Age of Intelligent Machines, Cambridge, MIT Press.

Kurzweil (R.), 1999, The Age of Spiritual Machines, Cambridge, MIT Press.

Kurzweil (R.), 2005, The Singularity is Near, New York, Viking.

LAMBert (Johann Heinrich), 2002, Nouvel Organon, phénoménologie, trad. franç. Gilbert Fanfalone, Paris, Vrin.

Landsberg (Peter), 2000, « Conflicting Arrows of Time », Nature, vol. 403, p. 609.

Latil (Pierre, de), 1953, La Pensée artificielle. Introduction à la cybernétique, Paris, Gallimard.

Mandelbrot (Jacques), 2007, «La pensée gestuelle dans la science comme dans l'art », Alliage, $\mathrm{n}^{\circ} 61$ (http://www.tribunes.com/tribune/alliage/61/page1/page1.html).

MAson (Samuel J.), 1953, «Feedback Theory - Some Properties of Signal Flow Graphs », Proceedings of the I.R.E., vol. 41, p.1144-1156.

Mason (S. J.), 1956, «Further Properties of Signal Flow Graphs », Proceedings of the I.R.E., vol. 44, p. 920-926.

McCulloch (Warren) et PitTs (Walter), 1943, «A Logical Calculus of the Ideas Immanent in Nervous Activity ", Bulletin of Mathematical Biophysics, vol. 5, p.115-133.

Menra (Jagdish), 1994, The Beat of a Different Drum. The Life and Science of Richard Feynman, New York, Clarendon Press.

Neumann (John von), 1996, Théorie générale et logique des automates, trad. franç. prés. par Gérard ChazAL, Seyssel, Champ Vallon.

Nicholson (Jeffrey), 2005, « The Meaning of Arrows: Diagrams and Other Facets in System Sciences Literature », Proceedings of the $38^{\text {th }}$ Annual Hawaï International Conference on Systems Sciences (HICSS'05), track 4, vol. 4, p. 99.2.

Øнгsтrøм (Peter), 1985, «W. R. Hamilton's View of Algebra as the Science of Pure Time and His Revision of This View ", Historia Mathematica, vol. 12, p. 45-55.

ONg (Walter J.), 1958, Ramus, Method and the Decay of Dialogue. From the Art of Discourse to the Art of Reason, Cambridge, Harvard University Press.

ONG (W. J.), 1977, Interfaces of the Word. Studies in the Evolution of Consciousness and Culture, Ithaca, Cornell University Press.

PASCH (Moritz), 1920-1921, «Der Ursprung des Zahlbegriffs », I. Archiv der Mathematik und Physik, vol. 28, 1920, p.17-34; II. Mathematische Zeitschrift, vol. 11, n¹-2, 1921, p.124-156.

PAsch (M.), 1927, Mathematik am Ursprung, Leipzig.

Preston-Dunlop (Valérie), 1968, « A Notation System for Recording Observable Motion », Int. J. Man-Machine Studies, vol. 1, p. 363-386.

Raeburn (Daniel), 2004, Chris Ware, Londres, Laurence King Publishing.

Robin (Léon), 1923, La Pensée grecque, Paris, La Renaissance du Livre (L'Évolution de l'Humanité). 
Russell (Bertrand) et Whitehead (Alfred. N.), 1910, Principia Mathematica, Cambridge, Cambridge University Press.

Schlain (Leonard), 1991, Art \& Physics, New York, William Morrow.

Suszko (Roman), 1958, «Syntactic Structure and Semantical Reference I», Studia Logica, vol. VIII, p. 213-244.

Triclot (Mathieu), 2008, Le Moment cybernétique. La constitution de la notion d'information, Seyssel, Champ Vallon (Milieux).

Turing (Alan), 1936, «On Computable Numbers with an Application to the Entscheidungsproblem », Proceedings of the London Mathematical Society, vol. 42, p. 230.

Wheeler (John A.) et Feynman (Richard Ph.), 1949, «Classical Elecrodynamics in Terms of Direct Interparticle Action », Review of Modern Physics, vol. 31, p. 425-433.

Whitenead (Alfred North), 1978, Process and Reality. An Essay in Cosmology, New York, Macmillan.

Wiener (Norbert), 1948, Cybernetics or Control and Communication in the Animal and the Machine, Paris/New York/Cambridge, Hermann/Wiley \& Sons/Technology Press.

WienER (N.), 1956, I am a Mathematician, New York, MIT Press.

Yates (Frances), 1975, L'Art de la mémoire, trad. franç. Daniel Arasse, Paris, Gallimard. 